Article

\title{
Hyperbolic Covariant Coherent Structures in Two Dimensional Flows
}

\author{
Giovanni Conti ${ }^{+}$(iD) and Gualtiero Badin *,+ (D) \\ Institute of Oceanography, Center for Earth System Research and Sustainability (CEN), University of Hamburg, \\ Bundesstrasse 53, 20146 Hamburg, Germany; giovanni.conti@uni-hamburg.de \\ * Correspondence: gualtiero.badin@uni-hamburg.de; Tel.: +49-040-428-385-656 \\ + These authors contributed equally to this work.
}

Received: 22 August 2017; Accepted: 21 September 2017; Published: 27 September 2017

\begin{abstract}
A new method to describe hyperbolic patterns in two-dimensional flows is proposed. The method is based on the Covariant Lyapunov Vectors (CLVs), which have the properties of being covariant with the dynamics, and thus, being mapped by the tangent linear operator into another CLVs basis, they are norm independent, invariant under time reversal and cannot be orthonormal. CLVs can thus give more detailed information about the expansion and contraction directions of the flow than the Lyapunov vector bases, which are instead always orthogonal. We suggest a definition of Hyperbolic Covariant Coherent Structures (HCCSs), which can be defined on the scalar field representing the angle between the CLVs. HCCSs can be defined for every time instant and could be useful to understand the long-term behavior of particle tracers. We consider three examples: a simple autonomous Hamiltonian system, as well as the non-autonomous "double gyre" and Bickley jet, to see how well the angle is able to describe particular patterns and barriers. We compare the results from the HCCSs with other coherent patterns defined on finite time by the Finite Time Lyapunov Exponents (FTLEs), to see how the behaviors of these structures change asymptotically.
\end{abstract}

Keywords: covariant Lyapunov vectors; dynamical systems; mixing; hyperbolicity; ergodic theory

PACS: 05.45.-a; 05.90.+m; 92.10.Ty; 92.10.Lq; 92.10.Lf; 47.20.De; 47.27.ed

\section{Introduction}

In the paradigm of chaotic advection, the trajectories of passive tracers can be complex even when the velocity field of the flow is simple. This is the case, for example, for time-dependent two-dimensional flows or even steady three-dimensional flows, like the celebrated ABCflow [1]. However, even flows with a complicated time-dependent structure allow for the formation of coherent patterns that influence the evolution of tracers. These structures are common in nature, appearing both at short and long time scales, as well as small and large spatial scales. Remarkable examples of these structures are eddies and jets in the ocean and atmosphere, the Gulf Stream current and ring clouds [2]. These patterns can influence, for example, the evolution of nutrients, as well as oil spills and other pollutants. Furthermore, these coherent structures could act as a local inhibitor for the energy transfer between scales [3]. They also appear for other planets and other astrophysical systems, such as the jets on the surface of Jupiter, Saturn and other gaseous planets and in the solar photospheric flows [4]. These structures, often referred to as Lagrangian Coherent Structures (LCSs), can shed light on the mixing and transport properties of a particular system on a finite time interval. The term "Lagrangian" in their name is motivated by the fact that they evolve as material lines with the flow [2]. A particular kind of LCS is called Hyperbolic LCS (HLCS) and can be seen as the locally most attracting or repelling material lines that characterize the dynamical system over a finite time interval. The term hyperbolic is just an analogy of the stability of fixed points in dynamical systems, since usually one wants to study 
non-autonomous systems for which entities such as fixed points or stable and unstable manifolds are not defined. Moreover, these systems are studied for finite time.

Early attempts to detect HLCSs were based on Finite Time Lyapunov Exponents (FTLEs), which measure the rate at which initial conditions (or equivalently, tracer particles) separate locally after a given interval of time [5-12]. One of the first rigorous definitions of the LCSs was based on ridges of the FTLEs [10]. FTLEs and the so-defined LCSs were used to study for example Lagrangian dynamics of atmospheric jets [13] and oceanic stirring by mesoscale eddies [14-19], describing for example the chaotic advection emerging by mixed layer instabilities and its sensitivity to the vertical shear [20]. A similar method to detect coherent structures uses the Finite Size Lyapunov Exponents (FSLEs), which represent the separation rate of particles given a specific final distance [21-23]. However, several counter examples are available for which both FTLE and FSLE ridges fail to characterize the LCSs [24,25]. Although the FTLE field remains a popular diagnostic of chaotic stirring, other methods now are available to detect LCSs, which include for example the so-called Lagrangian descriptors [26,27], which are based on integration along trajectories, for a finite time, of an intrinsic bounded positive geometrical and/or physical property of the trajectories themselves. Notice however that the method of Lagrangian descriptors is not objective [28]; regarding the connection between the Perron-Frobenius operator and almost invariant coherent sets of non-autonomous dynamical systems defined over infinite times [29], the use of braids [30] and the extrema of trajectories [31]. Other Fast Indicators (FIs) besides FTLEs and FSLEs, i.e., computational diagnostics that characterize chaos quickly and can be used to determine coherent structures, are the Smaller (SALI) and Generalized (GALI) Alignment Indices [32], the Mean Exponential Growth rate of Nearby Orbits (MEGNO) [33] and the Finite Time Rotation Number (FTRN) [34-36].

Particularly promising for the detection of coherent structures is a variational theory that considers the extremum properties of a specific repulsion rate function [25,37-42]. Using this theory, it has been further shown that HLCSs can be described using geodesics [43]. In this way, HLCSs can be represented as minimizers of a material length, with specific boundary conditions for the variation function. Another geodesic theory describes HLCSs in terms of the shearless transport barrier that minimizes the average shear functional [41,44]. These theories are based on the computation of shrink/stretch-lines (tensor lines); thus, trajectories along the eigenvectors of the deformation tensor are also called the Cauchy Green Tensor (CGT). Geodesic theories are also able to detect two other kinds of LCSs called parabolic and elliptic, which are however of no interest for the present work.

All these methods such as FTLEs, FSLEs or the variational and geodesic theories aim to find particular structures on the flow, among these the most repelling, or attracting, structures in the flow on a finite time interval, the HLCS. Particle tracers around these most influential material structures in finite time are maximally repelled or attracted. However, changing the finite time interval under study also changes the dynamical system and the correspondent structures emerging from the flow. Recent effort has been made in the understanding the instantaneous most influential coherent structures, Objective Eulerian Coherent Structures (OECSs), using a method that is not based on a finite time interval of evolution [45-47]. The tracer particles could be maximally repelled for a short time, but over a long time, they could have a different behavior. Is it natural to wonder what happens asymptotically to the tracer particles. Is it possible to find some coherent structures that suggest the asymptotic behavior of the tracer particles?

In this work, we thus propose an alternative method to detect coherent patterns emerging in chaotic advection, which is based on the Covariant Lyapunov Vectors (CLVs). CLVs were first introduced by Oseledec [48] and Ruelle [49], but for a long time, they have received very little attention due to the lack of an efficient algorithm to compute them. Only in the last decade has the computation of such vectors become possible [50-52], and CLVs have been used to investigate, e.g., the motion of rigid disk systems [53], convection [54] and other atmospheric phenomena [55-58]. For other theoretical discussions or for reviews on CLVs, see, e.g., [59-63]. Unlike the Lyapunov Exponents (LEs), which are time independent, the correspondent vectors, also known as forward and backward 
Lyapunov Vectors (LVs), do depend on time. The LVs are orthonormal, and their direction can thus give only limited information about the local structure of the attractor. LVs also depend on the chosen norm; they are not invariant under time inversion; and they are not covariant, where covariance is here defined as the property of the forward (backward) LVs to be mapped by the tangent dynamics in forward (backward) LVs at the image point [61]. Differently from the LVs, CLVs are norm independent, invariant for temporal inversion and covariant with the dynamics, making them thus mapped by the tangent linear operator into another CLV bases during the evolution of the system. They are not orthonormal, and their directions can thus better probe the tangent structure of the system.

All this intrinsic information can be summarized using the angle between the CLVs, a scalar field that allows one to investigate the spatial structures of the system. The need to pay more attention to the directions between LVs, backward and forward, has also been suggested for the study of turbulence [9] and for the definition of a diagnostic quantity for the study of mixing, Lyapunov's diffusion [64]. Using three simple examples, we show that the attracting and repelling barriers tend to align along the paths on which the CLVs are orthogonal. The directions of the CLVs along these maxima provide thus information on the attracting or repelling nature of the barriers and can be related to the geometry of the system. Using the CLVs, we can define structures, at a given time instant, that are asymptotically the most attractive or repelling. Furthermore, since these structures can be defined for every time instant, it is possible to follow the formation of coherent structures during the evolution of the flow.

In Section 2, we discuss the theory behind the CLVs and suggest a definition for coherent structures that give asymptotic information. The strategy will be to make use of the scalar quantity defined by the angle between the CLVs to locally identify the structures that asymptotically are maximally attractive or repulsive. In Section 3, we use the CLVs to identify particular patterns in three different systems, and we compare the results with FTLE fields. Finally, in Section 4, we summarize the conclusions.

\section{Covariant Lyapunov Vectors}

In this section, we summarize the theory behind CLVs for two-dimensional flows. For a more general and detailed review, see for example [61]. Let the open set $D \subset \mathbb{R}^{2}$ be the domain of the flow, $t \in \mathbb{R}$ the time and $\mathbf{v}(\mathbf{x}, t)$ a vector velocity field in $D$. The dynamical system that describes the motion of a tracer advected by the flow is thus:

$$
\begin{aligned}
& \frac{d \mathbf{x}\left(\mathbf{x}_{0}, t_{0} ; t\right)}{d t}=\mathbf{v}\left(\mathbf{x}\left(\mathbf{x}_{0}, t_{0} ; t\right), t\right), \\
& \mathbf{x}\left(\mathbf{x}_{0}, t_{0} ; t_{0}\right)=\mathbf{x}_{0},
\end{aligned}
$$

where $\mathbf{x}\left(\mathbf{x}_{0}, t_{0}, t\right) \in D$ is the trajectory of the tracer starting at the point $\mathbf{x}_{0}$ at time $t_{0}$. To Equation (1) is associated the flow map $\boldsymbol{\phi}_{t_{0}}^{t}\left(\mathbf{x}_{0}\right)$ :

$$
\begin{aligned}
& \boldsymbol{\phi}_{t_{0}}^{t}: D \rightarrow D, \\
& \mathbf{x}_{0} \rightarrow \mathbf{x}\left(\mathbf{x}_{0}, t_{0}, t\right),
\end{aligned}
$$

that maps the initial position $\mathbf{x}_{0}$ at time $t_{0}$ to the position $\mathbf{x}\left(\mathbf{x}_{0}, t_{0}, t\right)$ at time $t$. It should be noted that the dependence on the initial condition is very important here, since the vectors will be considered as a function of time and of the initial positions. In the following, the contracted form $\mathbf{x}=\mathbf{x}\left(\mathbf{x}_{0}, t_{0}, t\right)$ will be used.

At each point $\mathbf{x} \in D$, we can identify the tangent space $T_{\mathbf{x}} D \subset \mathbb{R}^{2}$. Infinitesimal perturbations, $\mathbf{u}(t) \in T_{\mathbf{x}} D$, to a trajectory of this system can be described by the linearized system:

$$
\begin{aligned}
& \frac{d \mathbf{u}(t)}{d t}=\mathbf{J}(t) \mathbf{u}(t), \\
& \mathbf{u}\left(t_{0}\right)=\mathbf{u}_{0},
\end{aligned}
$$


where $\mathbf{J}(t) \in \mathbb{R}^{2 \times 2}$ is the Jacobian matrix composed by the derivatives of the vector field $\mathbf{v}(\mathbf{x}, t)$ with respect to the component of the vector $\mathbf{x}$. Using the fundamental matrix $\mathbf{M}(t) \in \mathbb{R}^{2 \times 2}$, of Equation (3), that satisfies:

$$
\begin{aligned}
\frac{d \mathbf{M}(t)}{d t} & =\mathbf{J}(t) \mathbf{M}(t), \\
\mathbf{M}(0) & =\mathbf{I}
\end{aligned}
$$

we define the so-called tangent linear propagator:

$$
\mathbf{F}\left(t_{0}, t\right)=\mathbf{M}(t) \mathbf{M}\left(t_{0}\right)^{-1} .
$$

$\mathbf{F}\left(t_{0}, t\right)$ maps a vector in $\mathbf{x}_{0}$ at time $t_{0}$ into a vector in $\mathbf{x}$ at time $t$ along the same trajectory of the starting system Equation (1), that is:

$$
\mathbf{u}(t)=\mathbf{F}\left(t_{0}, t\right) \mathbf{u}\left(t_{0}\right)
$$

According to Equation (5), the propagator is always nonsingular. In terms of the flow map, the tangent linear propagator is:

$$
\mathbf{F}\left(t_{0}, t\right)=\nabla \boldsymbol{\phi}_{t_{0}}^{t} .
$$

Exploiting Oseledec's Theorem $[48,65]$, it is possible to characterize the system using quantities that are independent of $t$ or $t_{0}$. By virtue of this theorem, the far-future operator:

$$
\mathbf{O}^{+}\left(t_{0}\right)=\lim _{t \rightarrow+\infty}\left(\mathbf{F}\left(t_{0}, t\right)^{\top} \mathbf{F}\left(t_{0}, t\right)\right)^{1 / 2\left(t-t_{0}\right)}
$$

and the far-past operator:

$$
\mathbf{O}^{-}(t)=\lim _{t_{0} \rightarrow-\infty}\left(\mathbf{F}\left(t_{0}, t\right)^{-\top} \mathbf{F}\left(t_{0}, t\right)^{-1}\right)^{1 / 2\left(t-t_{0}\right)}
$$

are well-defined quantities. Note that the product $\mathbf{F}^{\top}\left(t_{0}, t\right) \mathbf{F}\left(t_{0}, t\right)$ determines the Euclidean norm of the tangent vectors in the forward-time dynamics (a similar role is played by $\mathbf{F}^{-\top}\left(t_{0}, t\right) \mathbf{F}^{-1}\left(t_{0}, t\right)$ for the backward-time dynamics), in fact,

$$
\|\mathbf{u}(t)\|=\left[\mathbf{u}\left(t_{0}\right)^{\top}\left(\mathbf{F}^{\top}\left(t_{0}, t\right) \mathbf{F}\left(t_{0}, t\right)\right) \mathbf{u}\left(t_{0}\right)\right]^{1 / 2} .
$$

Operator Equations (8) and (9) probe respectively the future and past dynamics of a certain point and share the same eigenvalues:

$$
\lambda_{1} \geq \lambda_{2}
$$

which, assuming ergodicity, are independent of time and space. Each eigenvalue has multiplicity $m_{i}\left(m_{1}+m_{2}=2\right)$. Their logarithms correspond to the LEs of the dynamical system Equation (1). If the limits in Equations (8) and (9) are not considered, the resulting eigenvalues are time and space dependent and are called FTLEs.

The two operator Equations (8) and (9) can be evaluated at the same point in space at a given time $t$. The correspondent eigenvectors, $\left\{\mathbf{l}_{1}^{+}(t), \mathbf{1}_{2}^{+}(t)\right\},\left\{\mathbf{l}_{1}^{-}(t), \mathbf{l}_{2}^{-}(t)\right\}$, will define thus the forward and backward Lyapunov basis computed at the same time. Conversely, the respective eigenvalues, whose bases are time dependent, depend on the chosen scalar product and are not invariant under time reversal. Furthermore, these vectors are always orthogonal and give thus limited information on the spatial structure of the configuration space. 
To overcome these issues, one can build particular spaces, the backward and forward Oseledec subspaces, defined as [48]:

$$
L_{i}^{-}(t)=\operatorname{span}\left\{\mathbf{l}_{j}^{-}(t) \mid j=1, i\right\}, \quad i=1,2, \quad L_{0}^{-}(t)=\varnothing,
$$

and:

$$
L_{i}^{+}(t)=\operatorname{span}\left\{\mathbf{1}_{j}^{+}(t) \mid j=i, 2\right\}, \quad i=1,2, \quad L_{0}^{+}(t)=\varnothing
$$

In the forward dynamics, the generic vector $\mathbf{1}_{i}^{+}(t)$ grows or decays exponentially with an average rate $\lambda_{i}$. If the system is evolved forward in time, by means of the tangent linear propagator, the evolution of the vector $\mathbf{l}_{1}^{+}(t)$ will have a non-zero projection inside the space generated by $\mathbf{l}_{1}^{+}\left(t^{\prime}\right)$, but it will also have a non-zero projection onto the space generated by $\mathbf{l}_{2}^{+}\left(t^{\prime}\right)$. On the other hand, $\mathbf{1}_{2}^{+}(t)$ will be transported onto the space generated by $\mathbf{l}_{2}^{+}\left(t^{\prime}\right)$ and will have zero projection onto the space generated by $\mathbf{l}_{1}^{+}\left(t^{\prime}\right)$. Repeating similar arguments for the backward Lyapunov Vectors leads to the observation that $L_{i}^{-}$and $L_{i}^{+}$are covariant subspaces,

$$
\begin{aligned}
& L_{i}^{-}\left(t^{\prime}\right)=\mathbf{F}\left(t, t^{\prime}\right) L_{i}^{-}(t), \\
& L_{i}^{+}\left(t^{\prime}\right)=\mathbf{F}\left(t, t^{\prime}\right) L_{i}^{+}(t) .
\end{aligned}
$$

Vectors that are covariant with the dynamics and invariant with respect to time reversal will be found at the intersection of the Oseledec subspaces, i.e., at:

$$
\mathbf{w}_{i}(t)=L_{i}^{-}(t) \cap L_{i}^{+}(t) .
$$

These spaces, often referred to as Oseledec splitting [48,49,51,63], are not empty [61], and their vectors, also called covariant Lyapunov vectors (CLVs), are covariant with the dynamics, i.e.,

$$
\mathbf{F}\left(t, t^{\prime}\right) \mathbf{w}_{i}(t)=\mathbf{w}_{i}\left(t^{\prime}\right)
$$

It should be noted that CLVs posses thus the properties of a semi-group. These vectors have an asymptotic grow the or decay with an average rate $\lambda_{i}$, so that their asymptotic behavior can be summarized as:

$$
\left\|\mathbf{F}(t, t \pm \tau) \mathbf{w}_{i}(t)\right\| \approx e^{ \pm \lambda_{i} \tau},
$$

where $\tau=\left|t^{\prime}-t\right|$, which shows their invariance under time reversal. Note also that these vectors do not depend on any particular norm.

Equations (12), (13) and (15) imply a simple relation between CLVs and LVs in a twodimensional system,

$$
\mathbf{w}_{1} \equiv \mathbf{1}_{1}^{-} \quad \text { and } \quad \mathbf{w}_{2} \equiv \mathbf{1}_{2}^{+},
$$

The first CLV corresponds to the first backward Lyapunov vector and the second CLV to the second forward Lyapunov vector.

Furthermore, if the Jacobian matrix appearing in Equation (3) is constant, the CLVs are not just covariant, but invariant with the dynamics. In this particular case, in fact, they correspond to the eigenvectors of the Jacobian, and the eigenvalues of this matrix coincide with the LEs.

Since the CLVs highlight particular expansion and contraction directions at each point of the coordinate space and these directions are not necessarily orthogonal, they can be used to understand the geometrical structure of the tangent space. This geometric information can be summarized by the scalar field of the angle $\theta(t)$ between the CLVs. Because the CLVs identify asymptotically the expansion and contraction direction sets of the tangent space associated at every point of the domain, the $\theta$ field represents a measure of the hyperbolicity of the system, that is a measure of the orthogonality between 
these two directions. Note that the orientation of the CLVs is defined as arbitrary. The angle between the CLVs,

$$
\theta(t) \in\left[0, \frac{\pi}{2}\right],
$$

is thus:

$$
\theta(t)=\cos ^{-1}\left(\left|\mathbf{w}_{1}(t) \cdot \mathbf{w}_{2}(t)\right|\right),
$$

where $\left\{\mathbf{w}_{1}(t), \mathbf{w}_{2}(t)\right\}$ are the first and the second CLVs [51]. It is interesting to point out that when $\theta(t)=\pi / 2$, the CLVs reduce to LVs in two dimensions, and the backward and forward Lyapunov bases coincide. If the computation of the angle is done at every point of the domain and if we consider the system Equation (1) in which the initial conditions are varied in such a way that all the domain is spanned, we can build a field of the orthogonality between the expansion and contraction directions of the system.

In the following section, we will show how CLVs, with their capability of probing the geometric structure of the tangent space, can be used to determine coherent structures that give asymptotic information on the tracer and, then, how the CLVs highlight the mixing template of the flow.

\section{Hyperbolic Covariant Coherent Structures}

Several frameworks are available to study passive scalar mixing. Although all these methods aim to describe the mechanism underlying the chaotic advection, they have significantly different approaches. However, most of them share a fundamental feature called objectivity. Objectivity is a fundamental requirement to define structures emerging from a flow. In practical applications, objectivity can be used, e.g., to move the reference frame into the reference frame of a coherent structure. In particular, a structure can be considered objective if it is invariant under a coordinate change of the form:

$$
\hat{\mathbf{x}}(t)=\mathbf{Q}(t) \mathbf{x}(t)+\mathbf{P}(t),
$$

where $\mathbf{Q}$ denotes a time-dependent orthogonal matrix and $\mathbf{P}$ a time-dependent translation [2]. The FTLEs, FSLEs and geodesic theory define objective quantities. Sala et al. [62] have shown that, generally, also for a linear transformation of coordinates, the new angle non-linearly depends on the angle of the old reference system. However, for the particular class of transformation, we are interested in, Equation (21), the angle $\theta$ is invariant. This means that structures highlighted by $\theta$ are objective.

To study the asymptotically most attractive or repelling behavior of tracer particles near a particular structure identified at a given instant of time, one can consider the lines $\mathbf{r}(s, t)$, with $s$ representing the length parameter, defined by:

$$
\begin{aligned}
& \mathbf{r}_{1}^{\prime}(s, t)=\mathbf{w}_{1}(\mathbf{r}(s, t), t), \\
& \mathbf{r}_{2}^{\prime}(s, t)=\mathbf{w}_{2}(\mathbf{r}(s, t), t),
\end{aligned}
$$

where the primes indicate the derivative with respect to $s$ and characterized by $\theta=\pi / 2$ along their paths. In fact, in this case:

$$
\theta(t)=\frac{\pi}{2} \Rightarrow \quad \mathbf{w}_{1} \| \mathbf{l}_{1} \quad \text { and } \quad \mathbf{w}_{2} \| \mathbf{l}_{2}
$$

where $\mathbf{l}_{1}$ and $\mathbf{l}_{2}$ are Lyapunov vectors. In these circumstances, the backward and the forward bases are coincident. A sphere of initial conditions around a point of the path will be deformed in an ellipsoid whose axis is aligned with these Lyapunov vectors. If Equation (22) is aligned along a ridge of the hyperbolicity field $\theta$, characterized by $\theta=\pi / 2$, then they are also pointwise the most attractive or repelling lines in terms of the asymptotic behavior of tracer particles. Consider in fact line Equation (22a) and its tangent CLV $\mathbf{w}_{1}$ as depicted in Figure 1. 


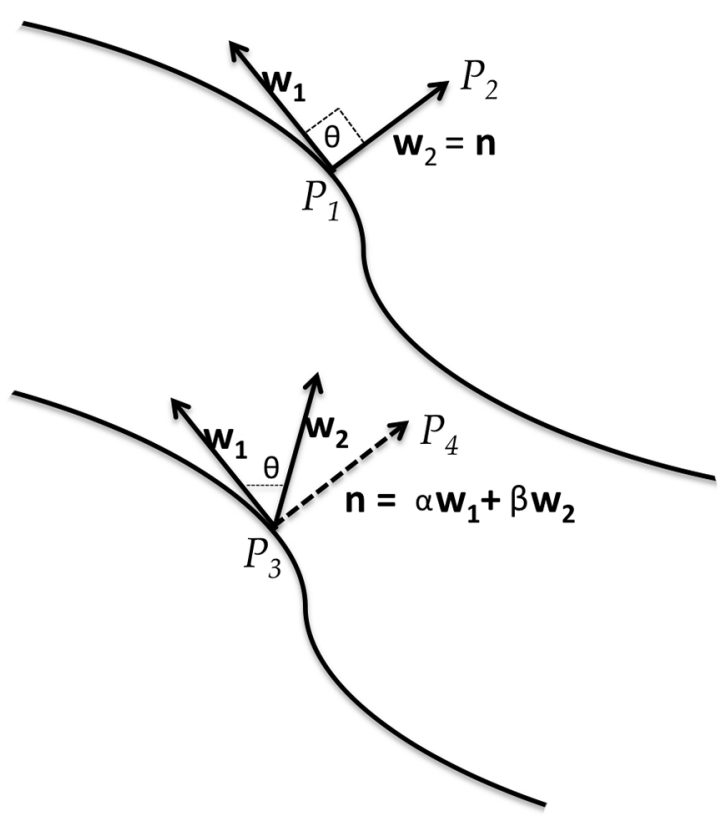

Figure 1. Orientation of Covariant Lyapunov Vectors (CLVs) on a most attracting material line (upper line) and on a neighboring material line (lower line). The distance $\left\|P_{2}-P_{1}\right\|$ decreases more quickly than $\left\|P_{4}-P_{3}\right\|$ since in the second curve, the normal vector connecting the points $P_{3}$ and $P_{4}$ is expressed as a linear combination of both CLVs.

Along this curve, for any point $P_{1}$, we can choose a point $P_{2}$, which is on the normal vector to the curve in $P_{1}, \mathbf{n}$. Because of Equation (11), the distance between these two points decreases as:

$$
\left\|P_{2}-P_{1}\right\| \propto\left\|\mathbf{w}_{2}\right\| \approx e^{\lambda_{2}\left(t^{\prime}-t\right)} .
$$

Consider now a point $P_{3}$ on a nearby curve and a point $P_{4}$ laying on the normal vector to the curve in $P_{3}$. The normal vector $\mathbf{n}$ at $P_{3}$ can be written as a linear combination of $\mathbf{w}_{1}$ and $\mathbf{w}_{2}$, and considering again Equation (11), one has thus:

$$
\left\|P_{4}-P_{3}\right\|=\left\|\alpha \mathbf{w}_{1}+\beta \mathbf{w}_{2}\right\| \approx e^{\lambda_{1}\left(t^{\prime}-t\right)}>e^{\lambda_{2}\left(t^{\prime}-t\right)} .
$$

The previous computation can be repeated analogously for $\mathbf{w}_{2}$. The same reasoning holds for $\mathbf{w}_{2}$. It must be remarked that Equation (22b) gives asymptotic information, but is defined for a particular instant of time. For every time instant, the line Equation (22), together with the orthogonality condition $\theta(t)=\pi / 2$ describe the so-called tensor lines and can be seen as the asymptotic version of shearless barriers [44].

Taking into account these properties of the CLVs, we propose a definition to describe these asymptotic coherent patterns:

Definition 1 (Hyperbolic Covariant Coherent Structures (HCCSs)). At each time t, a Hyperbolic Covariant Coherent Structure is an isoline of the hyperbolicity scalar field $\theta$ at the level $\theta=\pi / 2$. Its attractive or repelling nature is determined by the CLVs aligned with it.

It is important to emphasize the difference between the information provided by FTLEs and that provided by CLVs. FTLEs are the finite time version of Lyapunov's exponents and, as shown in the Appendix, are calculated as a mean time of the logarithms of the separation of two trajectories that 
start from nearby points. The FTLEs therefore give the average information over the time interval considered. Moreover, if the time interval is long enough and the analyzed region can be considered ergodic, the dependence on initial conditions is lost and, therefore, the possibility of displaying possible structures. CLVs, on the contrary, as well as LVs, depend on a time instant and do not converge to the same value. CLVs therefore allow the definition of instant structures, which, however, give asymptotic information.

This approach presents some numerical challenges: CLVs are not computed as quickly as the FTLEs, and numerically, in order to identify the isolines, it is necessary to consider an interval of values for the angle. The HCCSs are thus computed here considering the isoregion of $\theta \in\left[\frac{\pi}{2}-\delta, \frac{\pi}{2}\right]$, where $\delta$ is 0.087 rads.

\section{Numerical Examples}

The algorithm used in this work is the same presented in [51], so only a schematic of its structure will be presented here. This algorithm can compute a large number of CLVs converging exponentially quickly when invertible dynamics is considered. The basic idea is that, if the backward Lyapunov vectors basis $\left\{\mathbf{l}_{1}^{-}(t), \mathbf{l}_{2}^{-}(t)\right\}$ is evolved by means of the operator $\mathbf{F}$, it is always possible to keep it orthonormalized with a $Q R$ decomposition and store the corresponding upper triangular projection matrix. It is then possible to exploit the information contained in these upper triangular matrices to evolve backward in time arbitrary vectors that will converge to the CLVs [51]. This is done in five different phases, which are described in Appendix A.

In the next section, we investigate the hyperbolicity field $\theta$ for three different examples, which include one autonomous Hamiltonian flow and two non-autonomous two-dimensional flows. The HCCSs emerging from the angle between the CLVs are compared with the FTLE field, while their attractive or repelling nature will be discussed in terms of the CLVs. The algorithm for the search of the CLVs and the computation of the FTLEs explores the same dynamics, because it is used for the same time interval. However, the final CLVs are found just for a temporal subinterval of the whole time used: see Appendices A and B.

Technical details about the three different examples are included in Appendix B for a clearer description of the physical results in the following.

\subsection{A Simple Autonomous Hamiltonian System}

In this preliminary example, we investigate the HCCSs in an autonomous Hamiltonian flow map. The time-independent Hamiltonian, corresponding to the stream function of the flow, is:

$$
H=\frac{x^{2} y}{2}
$$

so that the dynamical equations of motion are:

$$
\begin{aligned}
& \frac{d x}{d t}=-\frac{x^{2}}{2}, \\
& \frac{d y}{d t}=x y,
\end{aligned}
$$

with $x\left(t_{0}\right)=x_{0}$ and $y\left(t_{0}\right)=y_{0}$. Integration of Equation (27) yields the trajectories of the system, shown in Figure 2a, with flow map:

$$
\boldsymbol{\phi}_{t_{0}}^{t}\left(x_{0}, y_{0}\right)=\left[\begin{array}{c}
x(t)=-\frac{2 x_{0}}{2+x_{0} t} \\
y(t)=y_{0}\left(1+\frac{x_{0} t}{2}\right)^{2}
\end{array}\right] .
$$




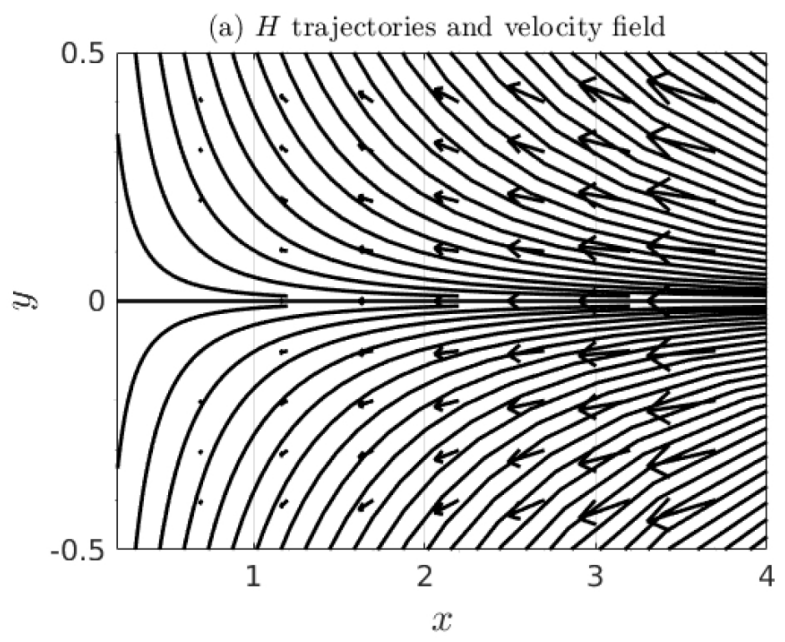

(b) Double gyre streamlines $t=5$

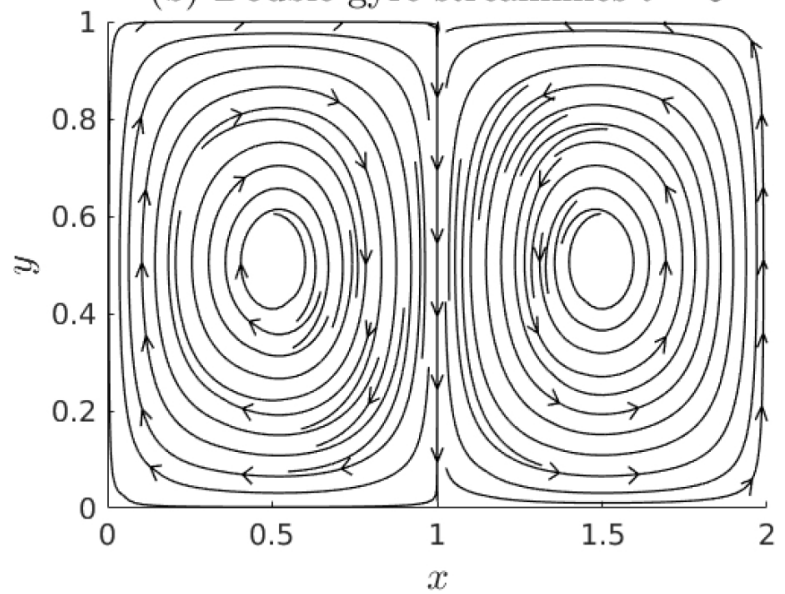

(c) Bickley jet streamlines $t=1.89 L_{x} / U$

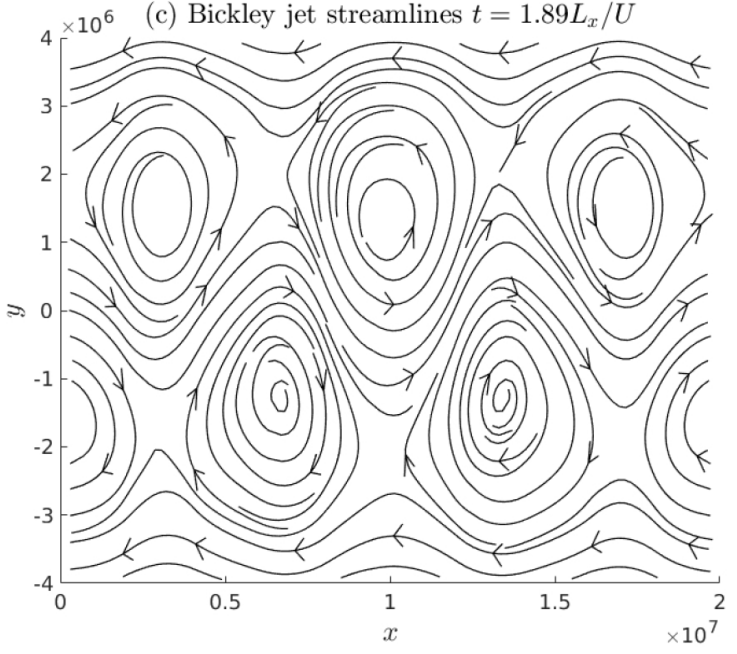

Figure 2. (a) Trajectories of the Hamiltonian system Equation (28) (solid line). The trajectory $y=0$ represents a repulsive barrier for the system. Notice that the arrows indicate the flow velocity field, which is expressed on the right-hand side of Equation (27), and not the CLVs, which are shown separately. $(\mathbf{b}, \mathbf{c})$ Streamlines for the double gyre and the Bickley jet, respectively.

Only the positive $x>0$ axis is considered, as the solution on the negative part blows up in finite time. As Figure $2 \mathrm{a}$ shows, the $y=0$ axis clearly divides the dynamics of the system into two different 
regions. There is no flux across this line, and the positive and negative $y$ regions are completely separated at each time. Furthermore, $y=0$ is the only material repelling line. Notice that in the figure, the arrows do not indicate the CLVs, but are just indicators of the magnitude of the flow. The trajectory $y=0$ behaves thus exactly as a material barrier dividing the system into two different regions, remains coherent at all times and repels every close trajectory. We apply the CLV theory to see if the angle between that vectors is able to detect such a kind of structure.

Figure 3a shows the maximum FTLE field computed in the time interval $t=[5,10]$ using definition Equation (A1). This field, referring to the initial grid conditions, does not highlight the $y=0$ barrier. Notice that if a bigger domain in the $y$ direction would have been selected, the FTLE field would have shown a large-scale modulation with larger values aligned along $y=0$. Figure $3 \mathrm{~b}$ shows the distribution of the CLVs angles at $t=5$. It is important to stress the fact that the FTLEs depend on a finite time interval, while CLVs, and then $\theta$, on a particular instant of time. The hyperbolicity field clearly shows values close to $\pi / 2$ along the $y=0$ axis. The direction of the first (red) and second (blue) CLV is shown for an enlargement of a region close to this structure in Figure 3c. It is visible that the second CLV, which characterizes the contraction direction, aside from numerical fluctuations, is aligned with the $\theta=\pi / 2$ structure, indicating a contraction direction and thus a repelling structure, i.e., a barrier, forward in time. Notice also that near $x=0$, the velocity field is close to zero, so that the barrier there is not well defined. This suggests that the dynamics near the origin evolves with a different time scale.

Along the $x$ axis, $\theta=\pi / 2$ remains constant for all times. Consider in fact the propagator Equation (7):

$$
\mathbf{F}\left(t, t^{\prime}\right)=\left[\begin{array}{cc}
1 / B^{2} & 0 \\
B t^{\prime} y_{0} & B^{2}
\end{array}\right]
$$

where:

$$
B=1+t^{\prime} x_{0} / 2
$$

Along $y=0$, both the tangent linear propagator:

$$
\mathbf{F}\left(t, t^{\prime}\right)=\left[\begin{array}{cc}
1 / B^{2} & 0 \\
0 & B^{2}
\end{array}\right]
$$

and the CGT (A2):

$$
\mathbf{C}\left(t, t^{\prime}\right)=\left[\begin{array}{cc}
1 / B^{4} & 0 \\
0 & B^{4}
\end{array}\right], \quad y_{0}=0,
$$

are diagonal. If at time $t$, one has $\theta(t)=\pi / 2$ for $y=0$, then:

$$
\mathbf{w}_{1}(t)=\xi_{1}=\left[\begin{array}{l}
0 \\
1
\end{array}\right] \text { and } \mathbf{w}_{2}(t)=\xi_{2}=\left[\begin{array}{l}
1 \\
0
\end{array}\right],
$$

where $\xi_{1}$ and $\xi_{2}$ are the eigenvectors of the CGT, and at time $t^{\prime}>t$ :

$$
\begin{aligned}
\cos \left(\theta\left(t^{\prime}\right)\right) & \propto \mathbf{F}\left(t, t^{\prime}\right) \mathbf{w}_{1}(t) \cdot \mathbf{F}\left(t, t^{\prime}\right) \mathbf{w}_{2}(t) \\
& =\mathbf{w}_{1}(t) \cdot \mathbf{C}\left(t, t^{\prime}\right) \mathbf{w}_{2}(t) \\
& =0 .
\end{aligned}
$$

This shows that $y=0$ is a repelling HCCS. 

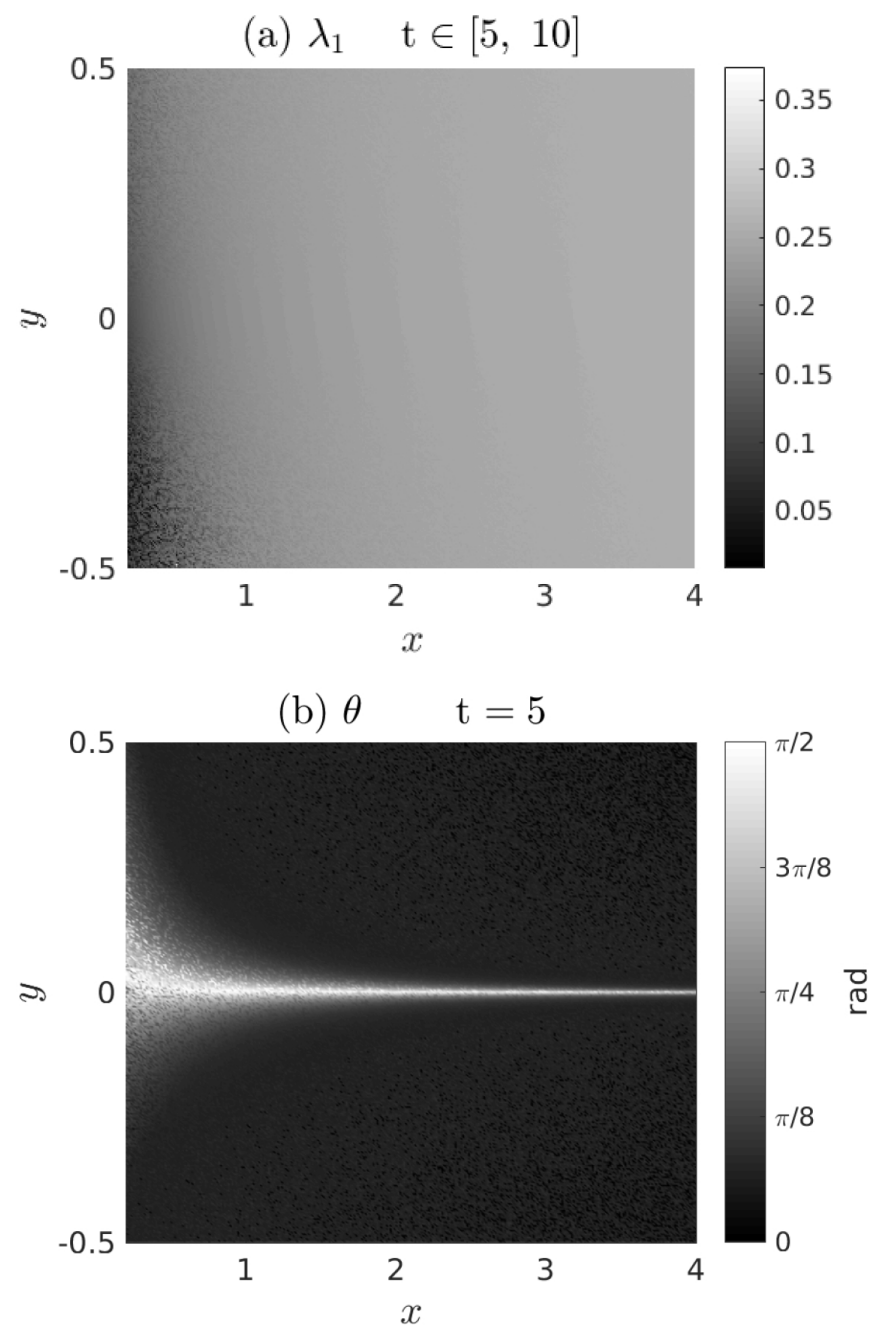

(c) CLVs map $\mathrm{t}=5$

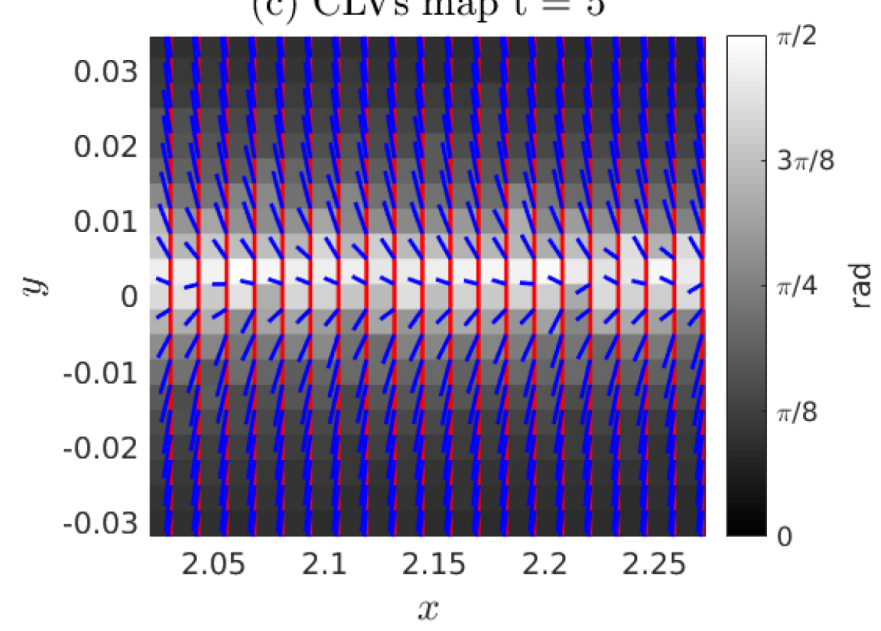

Figure 3. Finite Time Lyapunov Exponents (FTLEs) and CLVs fields for the Hamiltonian system Equation (27). (a) Maximum FTLE field computed from Equation (A1); (b) $\theta$ field; (c) zoom of the domain to show the CLV fields. Arrows are not used due to the arbitrariness of the orientation of the CLVs. The red lines are associated with $\mathbf{w}_{1}$, the expansion direction, while the blue lines are associated with $\mathbf{w}_{2}$, the contraction direction. 


\subsection{Double Gyre}

The previous analysis is now applied to a time-dependent system called double gyre flow $[10,66]$, which is a simplification of observed geophysical flows consisting of two counter rotating vortices that expand and contract periodically. The tracers moving in this flow satisfy the following dynamical equations:

$$
\begin{aligned}
& \frac{d x}{d t}=-\pi A \sin (\pi f(x, t)) \cos (\pi y) \\
& \frac{d y}{d t}=\pi A \cos (\pi f(x, t)) \sin (\pi y) \frac{d f(x, t)}{d x}, \\
& f(x, t)=\epsilon \sin (\omega t) x^{2}+(1-2 \epsilon \sin (\omega t)) x
\end{aligned}
$$

with initial conditions $x(0)=x_{0}, y(0)=y_{0}$. The streamlines of the flow at $t=5$ are shown in Figure 2b.

We consider two numerical experiments, $D V 1$ and $D V 2$, in which the CLVs are computed in two different time intervals with a non-null intersection. During DV1, we compute the CLVs in the interval $t=[5,10]$ and during $D V 2$ for $t=[10,20]$. It is important, as will be shown in this section, to highlight that $\theta$ is a quantity that depends on a particular time instant and not on the time interval considered for its computation, as long as the interval considered is sufficiently long. Figure 4 shows a comparison between the angle $\theta$ and the FTLEs for this system. The first column is relative to $D V 1$, while the second column is relative to $D V 2$. The $\lambda_{1}$ fields, the maximum FTLEs, are shown in Figure $4 \mathrm{a}, \mathrm{b}$ for $D V 1$ and $D V 2$, respectively. The regions characterized by white color correspond to the maximum exponential growth rate. Ridges of these regions correspond to the LCSs of the system according to the definition by Shadden et al. [10]. It is interesting to notice how, although in Figure $4 b$, the ridges of the FTLEs are more developed, the $\lambda_{1}$ field converges to the same structures, and the maximum values do not change. The FTLEs give the overall information of the stretching and folding over the whole time interval considered, but they cannot say anything about the state of the coherent structures at a particular instant of time. Figure $4 \mathrm{c}, \mathrm{d}$ show the hyperbolicity field $\theta$ computed for a particular time instant. The white color here represents the maximum values of the hyperbolicity of the system, which means $\theta=\pi / 2$, and highlight possible HCCSs as defined in Definition 1. Although the FTLEs and $\theta$ fields exhibit similar structures, it is interesting to point out that the ridges of the FTLEs do not necessarily correspond to the regions of maximum hyperbolicity. Near the central regions of the two vortices, the hyperbolicity field has a noisy appearance, due o the fact already discussed that in there the expansion and contraction, the directions are not well defined, and the CLVs become tangent to each other. Notice that this effect does not influence the detection of hyperbolic regions. Figure $4 \mathrm{e}, \mathrm{f}$ highlights the differences between FTLEs and the $\theta$ fields shown respectively for the two experiments $D V 1$ and DV2. As already mentioned, high values of the FTLEs do not necessarily correspond to high values of the $\theta$ field. The joint probability plots underline the fact that in general, there is not a one-to-one relation between the $\theta$ and FTLE fields. In particular, small values of FTLEs are related to the broadest range of possible values for the angle between CLVs. This can be understood considering that the FTLEs measure the exponential growth rate of the divergence of nearby trajectories. Where the FTLEs are smaller, the expansion and contraction directions are not well defined, and a broader range for the possible values of the angle is obtained. Although there is not a clear relation between the two quantities, it is interesting to point out that, for this example, the maximum values of FTLEs correspond to a reduced range of possible values of $\theta$, and for the $D V 2$ experiment, there is a univocal correspondence. The joint probabilities are asymmetric, and their peaks towards the highest values of $\theta$ highlight the fact that the system is hyperbolic. In this case, in fact, it is possible to find, almost for every point of the domain, well-defined expansion and contraction directions. It is interesting to note that the joint probability of Figure $4 \mathrm{f}$ is less asymmetric with respect to the correspondent joint probability in Figure 4e. 

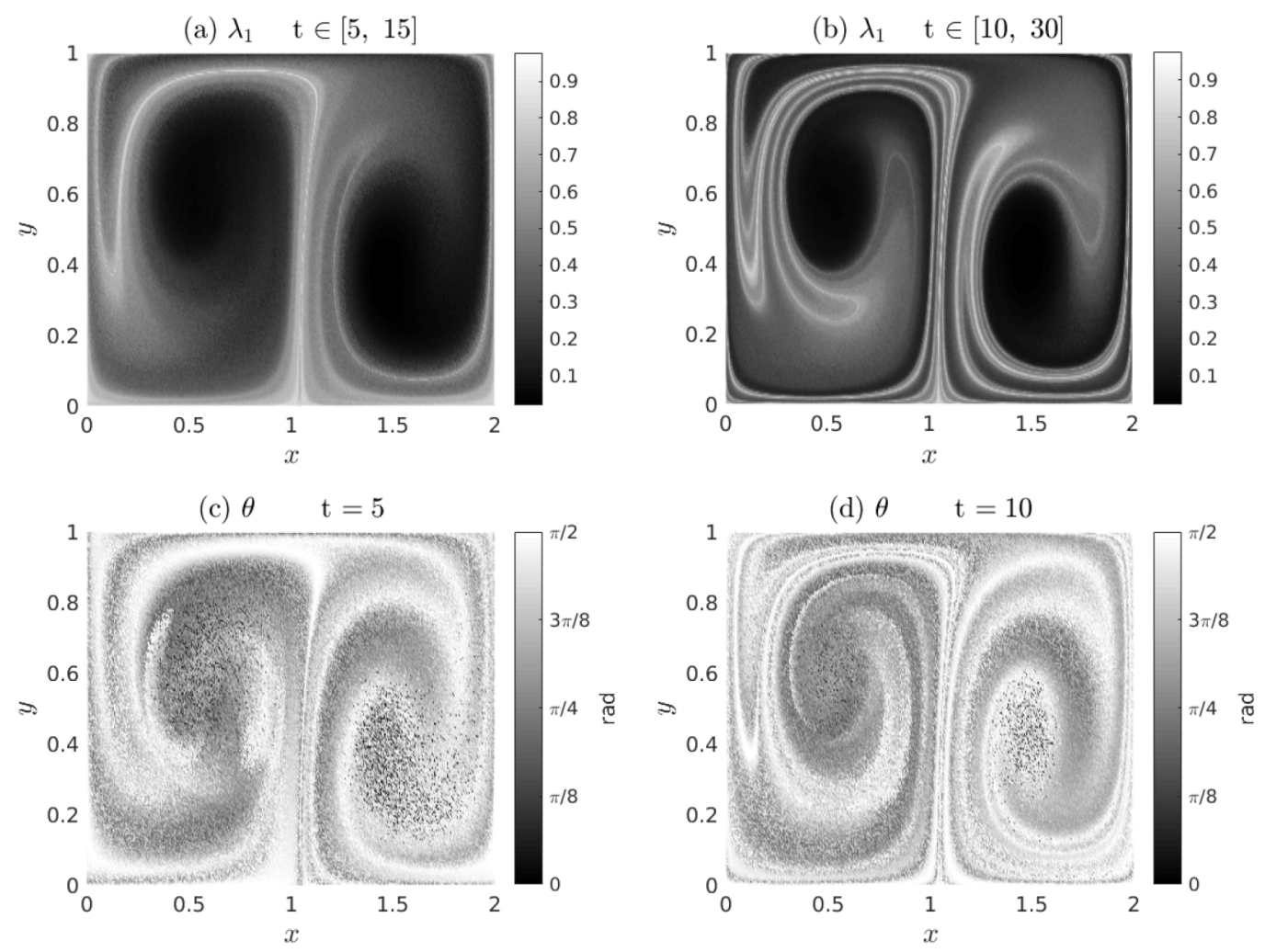

(e)
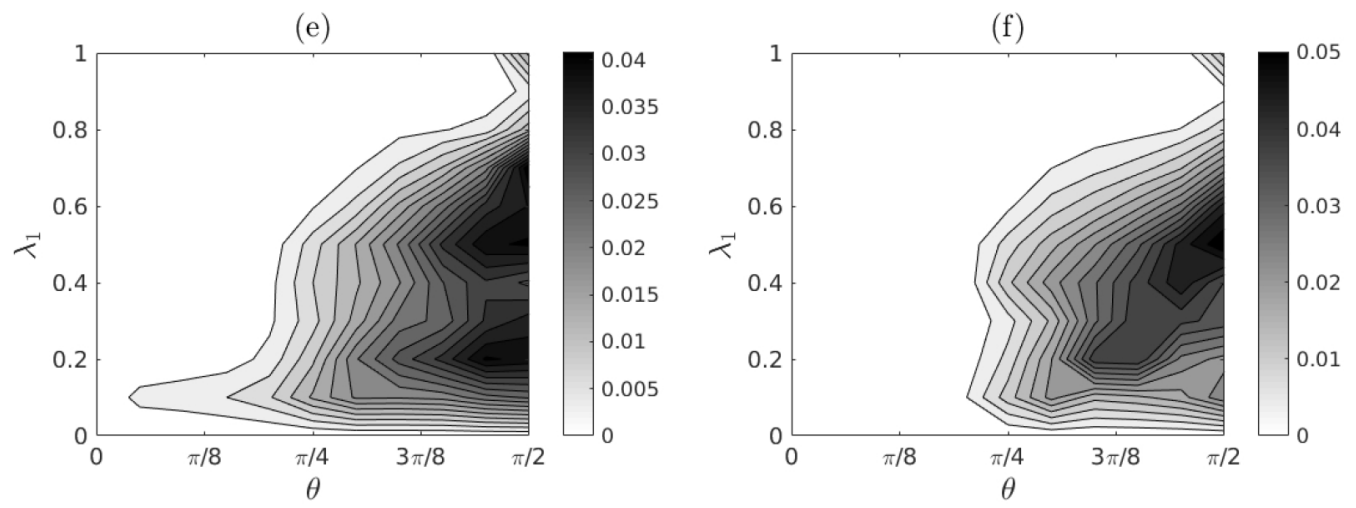

Figure 4. FTLEs, CLVs and joint probability plot for the experiments DV1 (left panels) and DV2 (right panels) of the double gyre system. (a,b) show the maximum FTLE fields computed from (A1); $(\mathbf{c}, \mathbf{d})$ show the angle between the CLVs for the two experiments; $(\mathbf{e}, \mathbf{f})$ show the joint probability between FTLEs and the angles shown in the previous panels.

Figure 5 compares the HCCSs at time $t=10$ for the experiment $D V 2$, found with Definition 1 , and the FTLE field shown in Figure $4 \mathrm{~b}$. In the right panel, there is a blow up of a region containing HCCSs. These pictures show that the instant structures highlighted by the HCCSs do not always correspond to the ridges of the FTLEs. The contours of the regions characterized by $\theta=\pi / 2$ define the shapes of HCCSs, but to understand their attractive or repelling nature, it is necessary to look at the CLVs that characterized those regions. The right panel of Figure 5 shows the repelling nature of the HCCSs in the zoomed region, since the contour is aligned with the second CLV, $\mathbf{w}_{2}$, which defines the contraction direction. Every particle of the tracer near these HCCSs will tend asymptotically to move away from them. 


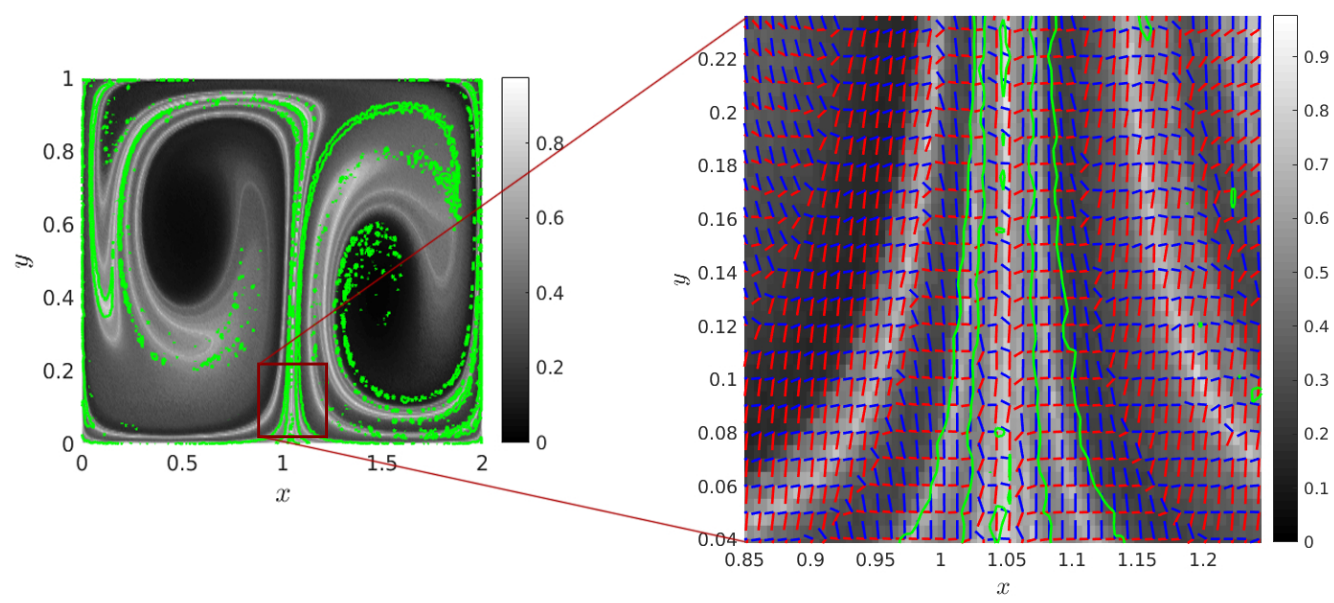

Figure 5. Comparison between the Hyperbolic Covariant Coherent Structures (HCCSs) at time $t=5$, green lines, computed for the experiment DV2 and the FTLE field shown in Figure $4 \mathrm{~b}$. In the right panel there is a zoom of a portion of the domain in which are also shown the CLVs characterizing that region. The blue direction is contracting, while red is expanding. Since the contour in this region is aligned with the second CLVs, the blue one, the character of the HCCSs here is repulsive.

Figure 6 compares the evolution of the $\theta$ field in $D V 1$, computed at $t=10$, with the angles computed at the same time for the experiment $D V 2$. The region of maximum hyperbolicity that appears in Figure 6a corresponds to the same region that is found in Figure 4d. The difference between the two fields (Figure 6b) shows large values in regions with low hyperbolicity and values close to zero for $\theta=\pi / 2$, implying a convergence of the $\theta$ field. It should also be noted that the structure highlighted by the maximum hyperbolicity in the left part of the domain is pretty similar to the one shown in Figure 4a. Once again, this underlines that the FTLE field gives that overall information and not an instantaneous one. The HLCSs found by using the FTLEs are, by definition, strongly related to the time interval chosen for the study, while the HCCSs, which correspond to instantaneous structures of maximum hyperbolicity, are independent of the time interval considered for their computation, as shown in these two experiments. The short-term behavior of a passive tracer analyzed by HLCSs is strongly influenced by the time window used with respect to long-term behavior highlighted by HCCSs. It should also be noted that the HLCSs, once they have been found, are advected with the flow so as to ensure that they act as barriers for the passive tracer. However, this precludes the study of any new barriers that may arise in the flow in a time subinterval. These constraints are not present in the long-term study performed with HCCSs.

It is also interesting to consider the evolution of the PDF of the hyperbolicity field, $P(\theta, t)$. If the distribution is peaked around $\pi / 2$, the expansion and contraction directions are almost everywhere perpendicular between each other. If the distribution is peaked around zero, the expansion and contraction directions are almost tangent everywhere. If the distribution is flat, there is not a clear correlation between the expansion and contraction directions. Figure 7 shows the PDF of $\theta$ computed for the initial and final time of the CLVs' computation for the experiment DV2. The final PDF (full line) highlights an increasing of points with height hyperbolicity and an increment of points in the tail of the distribution near zero, with respect to the PDF computed at the beginning of the interval (dashed line). During the evolution in the interval $t=[10,20]$, the distribution becomes more asymmetric. The change in shape is visible also looking at the first four moments of the PDF computed at every time step in this time interval (Figure 8). All the moments of the distribution display only small changes in time during the evolution. This time interval is characterized by a mean value of $\theta$ oscillating in the range [1.20, 1.23]. A monotonic change in the mean, possibly given by mixing, is visible after $t \approx 16$. It should be noted however that the range of this change 
is very small. The s.t.d.of the angles distributions is characterized by values that change within the interval $[0.25,0.28]$. After $t \approx 16$, the s.t.d. shows a monotonic increase, as a signature that, if the change in time is given by mixing, this must be of an adiabatic nature and thus not resulting in a homogenization of the field. The third moment of the PDF decreases in time from -0.57 to -0.91 , showing thus a negatively-skewed distribution. Finally, the kurtosis initially increases from 2.81 to 4.03 and then shows a slight decrease in values, generally indicating a less flat distribution than the normal distribution. Although the motion of the velocity field is periodic, the motion of the tracers in this flow is chaotic. This is the reason why we do not see in this interval a periodicity in the moments of the PDF for the variable $\theta$.

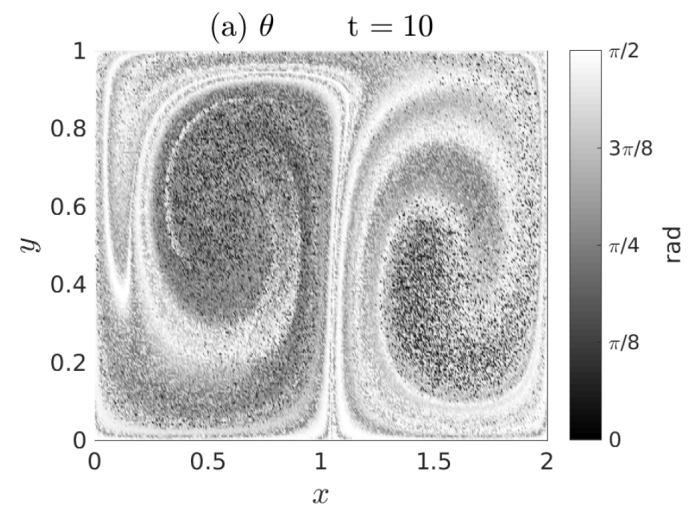

(b) $\theta$ differences

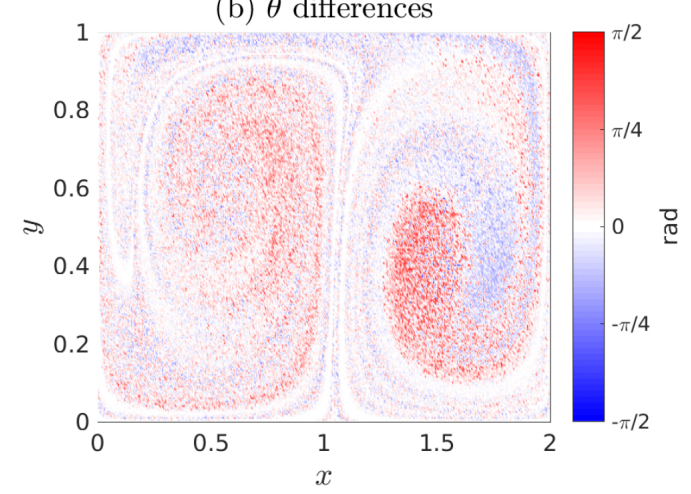

Figure 6. Comparison of the angle between CLVs, $\theta$, computed for the two experiments $D V 1$ and $D V 2$ at the same time $t=10$. (a) shows the evolution of the angle obtained in $D V 1$ at $t=10$; (b) shows the difference between the angle computed at the beginning of the experiment $D V 2$ and the one computed at the end of the interval of the CLVs computation for the experiment DV1.

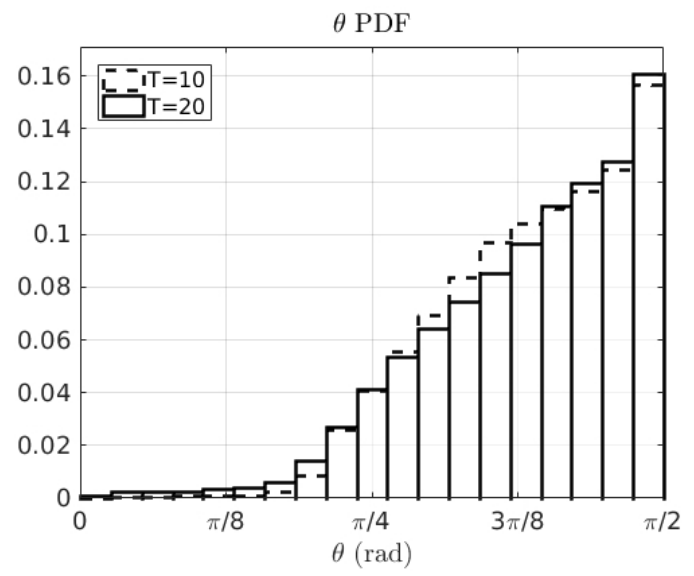

Figure 7. PDF of $\theta$ for the $D V 2$ experiment computed at $t=10$ (dashed line) and at $t=20$ (full line). 

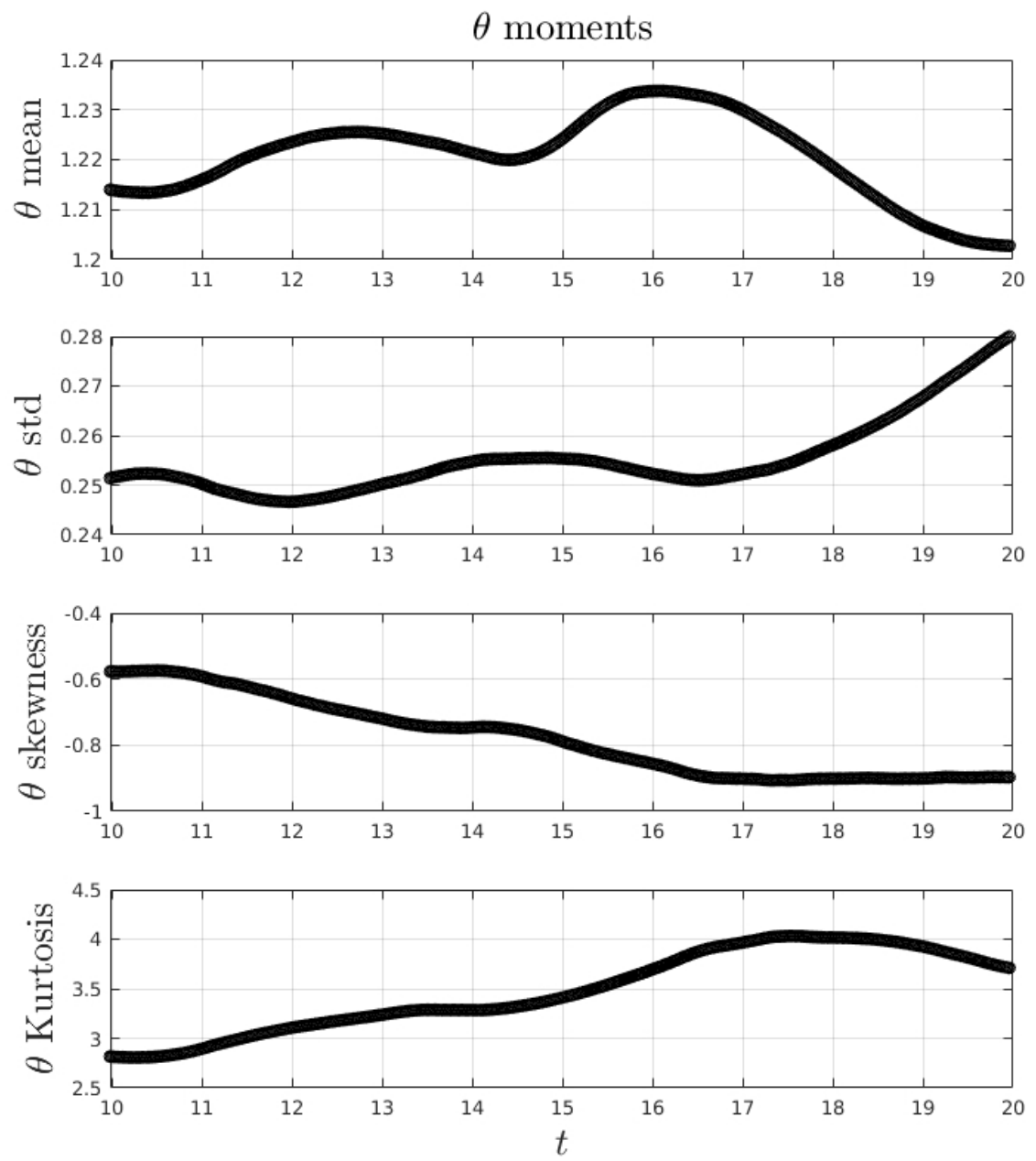

Figure 8. Evolution of the first four moments of the PDF for $\theta$ for the DV2 experiments in the interval $T 2=\left[\begin{array}{ll}10 & 20\end{array}\right]$.

\subsection{Bickley Jet}

The Bickley jet is an idealized model of a jet perturbed by a Rossby wave [66-68]. The velocity field is given in terms of the stream function, $\psi(x, y, t)$, which can be decomposed as the sum of a mean flow $\psi_{0}(x, y, t)$ and a perturbation $\psi_{1}(x, y, t)$ :

$$
\psi(x, y, t)=\psi_{0}(x, y)+\psi_{1}(x, y, t)
$$

where:

$$
\psi_{0}(x, y)=c_{3} y-U L_{y} \tanh \left(\frac{y}{L_{y}}\right)+\epsilon_{3} U L_{y} \operatorname{sech}^{2}\left(\frac{y}{L_{y}}\right) \cos \left(k_{3} x\right)
$$

and:

$$
\psi_{1}(x, y, t)=U L_{y} \operatorname{sech}^{2}\left(\frac{y}{L_{y}}\right) \mathcal{R} e\left[\sum_{n=1}^{2} \epsilon_{n} f_{n}(t) e^{i k_{n} x}\right] .
$$


Following the work by Onu et al. [66], the forcing is chosen as a solution that runs on the chaotic attractor of the Duffing oscillator:

$$
\begin{aligned}
& \frac{d \varphi_{1}}{d t}=\varphi_{2} \\
& \frac{d \varphi_{2}}{d t}=-0.1 \varphi_{2}-\varphi_{1}^{3}+11 \cos (t) \\
& f_{1}(t)=f_{2}(t)=2.62510^{-2} \varphi_{1}\left(t / 6.238 \times 10^{5}\right) .
\end{aligned}
$$

From now on, the time will be scaled with the quantity $L_{x} / U$. The streamline of the flow at $t=1.89$ is shown in Figure 2c.

As for the double gyre, we show two experiments, $B J 1$ and $B J 2$, for which the end of the CLVs' computation in the first experiment corresponds with the first CLV's computation for the second experiment.

The first column of Figure 9 shows the analysis for BJ1 and the second column for BJ2. Figure $9 \mathrm{a}, \mathrm{b}$ shows the field of the maximum FTLEs, $\lambda_{1}$. Results show the convergence for the FTLE fields. Maximum values of $\lambda_{1}$ are reached in the central jet and around the boundaries of the vortices. Figure $9 \mathrm{c}, \mathrm{d}$ shows the angle between CLVs, where the white color indicates $\theta=\pi / 2$. The $\theta$ fields clearly provide more details of the flow patterns than the FTLE fields. In agreement with the FTLE fields, values of $\theta$ close to $\pi / 2$ are present in narrow bands in the central jet, in the center and around the vortices. The angle field shows however that not all of the central band of the FTLE field represents an HCCSs. The $\theta$ fields show also spiraling patterns within the vortices, which are instead not visible in the FTLE fields. The spiral patterns are particularly evident in the upper vortices, where the perturbation $\psi_{1}$ acts as a positive feedback to the flow. In the bottom vortices, the perturbation acts instead to weaken the flow. Figure 9e,f shows the joint probability plots of the angles and the FTLEs just presented. These plots show that there is no clear relation between FTLEs and angle. To a given FTLE correspond many values of the angle between CLVs. As for the double gyre example, smaller values of the FTLEs correspond to the maximum range of possible values for $\theta$. However, differently from the double gyre, also high values of FTLEs correspond to a broad range of $\theta$ values. This can be explained considering that almost all the points corresponding to the highest values of FTLEs are contained in the central jet. In this region, the FTLEs converge to the same value, independently of the space position along the jet or time. Due to this convergence, we do not have much information about smaller structures that can characterize the system in the jet region as instead shown by the $\theta$ field. For this reason, the joint probability plots do not exhibit a reduced range of $\theta$ values in correspondence with the highest FTLEs. The comparison between the BJ1 and BJ2 integrations shows that for the Bickley jet, both the PDFs of the FTLE and $\theta$ field are more stationary in time with respect the double gyre.

The left panel of Figure 10 shows the superposition of HCCSs computed for $t=3.79$ for the experiment $B J 2$, green lines, and the FTLEs computed in the same experiments (Figure $9 \mathrm{~b}$ ). This figure points out about the fact that the central jet is not completely an HCCS. HCCSs are also present at the border of the vortices and in the center part of the upper vortices. The right panel of Figure 10 shows an enlargement of a portion of the central jet containing HCCSs and the CLVs in that part of the domain. In this picture, it is possible to appreciate the repelling nature of the HCCSs looking at the alignment of the contour with the second CLV. It is interesting to point out that, looking at the FTLE field, it is not possible to see ridges in the central jet. In this case, it is not possible to find LCSs, if we use the definition of LCSs based on the FTLE field [10,37], and this once again points out the the difference between the HCCSs and LCSs. 

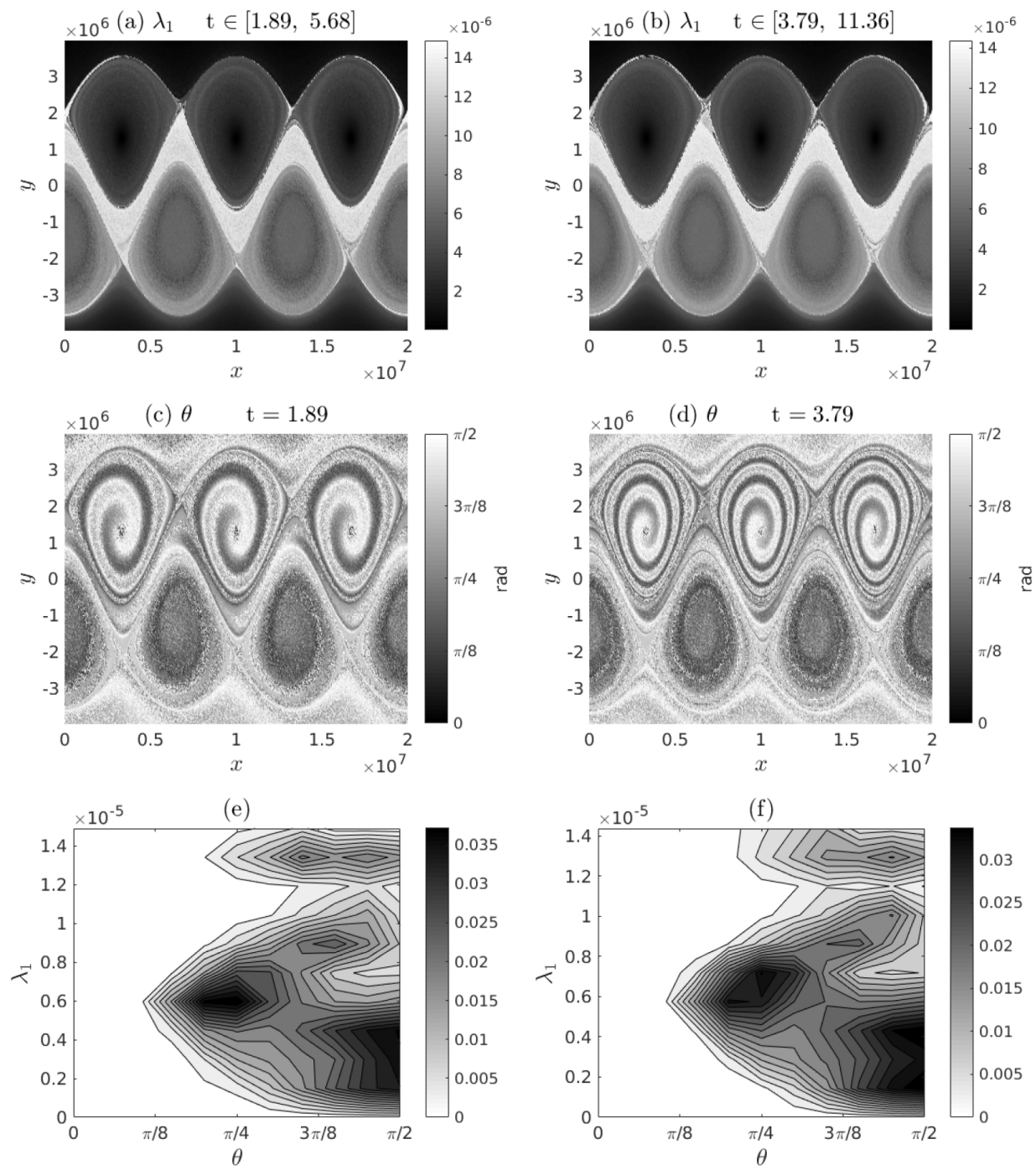

Figure 9. FTLEs, CLVs and joint probability plot for the experiments BJ1 (left panels) and BJ2 (right panels) of the Bickley Jet. (a,b) show the maximum FTLE fields computed from (A1); (c,d) show the angle between the CLVs for the two experiments; $(\mathbf{e}, \mathbf{f})$ show the joint probability between FTLEs and the angles shown in the previous panels.

Figure 11 shows the comparison between the angles at the end of the CLVs' computation interval of the experiment $B J 1$ with the one computed at the beginning of the CLVs' calculation interval for the $B J 2$ experiments. As for the double gyre, the comparison between Figure 11a and Figure 9d shows that regions characterized by $\theta=\pi / 2$, i.e., the HCCSs, have the same structure. The difference between the two fields (Figure 11b) shows that these regions are characterized by smaller errors. In contrast, the regions with low hyperbolicity of Figure 11a appear to be more noisy with respect to the same regions computed for the BJ2 simulation (Figure 9d). In agreement with this, the difference between the two fields shows that the low hyperbolicity regions are characterized by larger errors (Figure 11b).

Figure 12 shows the PDF of $\theta$ for the experiment BJ2 at the beginning and at the end of the CLVs computational interval. Results show that the PDF of the angle for this this interval remains stable. This is confirmed by the analysis of the moments of the distribution (not shown), which appear to be constant in time. With respect to the double gyre, the PDF for the Bickley jet is flatter, indicating the presence of a larger number of points in which the CLVs are not orthogonal. 


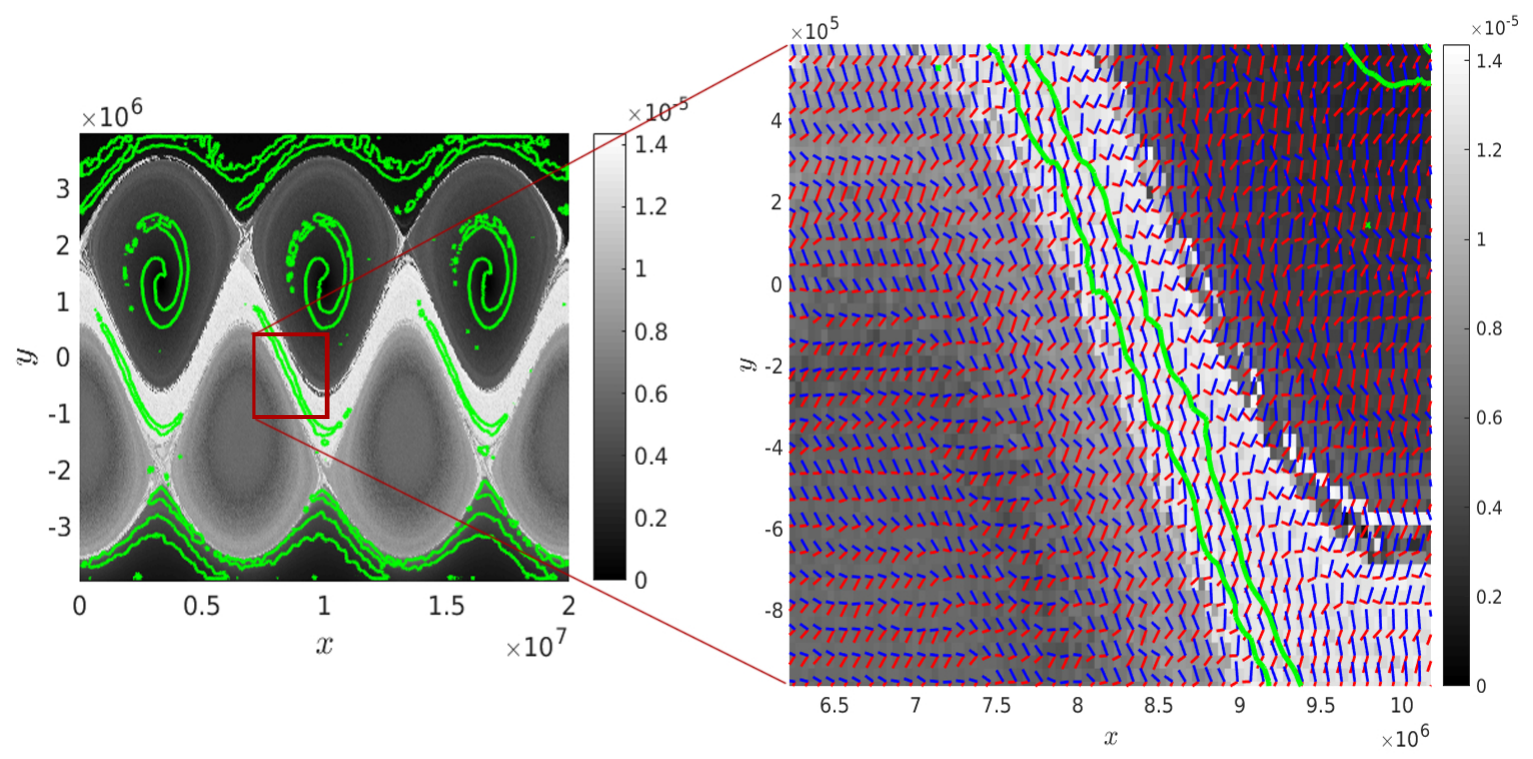

Figure 10. Comparison between the Hyperbolic Covariant Coherent Structures (HCCSs) at time $t=3.79$, green lines, computed for the experiment BJ2 and the FTLE field shown in Figure 9b. In the right panel there is a zoom of a portion of the domain in which are also shown the CLVs characterizing that region. The blue direction is contracting, while red is expanding. Since the contour in this region is aligned with the second CLVs, the blue one, the character of the HCCSs here is repulsive.
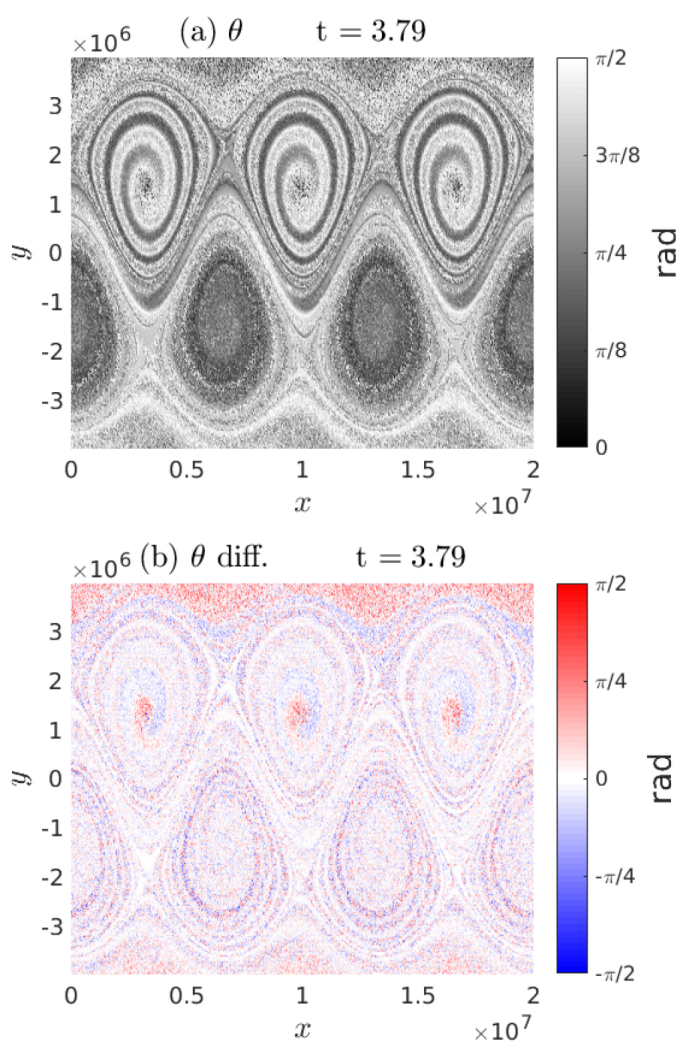

Figure 11. Comparison of the angle between CLVs, $\theta$, computed for the two experiments $B J 1$ and $B J 2$ at the same time $t=3.79$. (a) shows the evolution of the angle obtained in $B J 1$ at $t=3.79$; (b) shows the difference between the angle computed at the beginning of the experiment $B J 2$ and the one computed at the end of the interval of the CLVs computation for the experiment BJ1. 


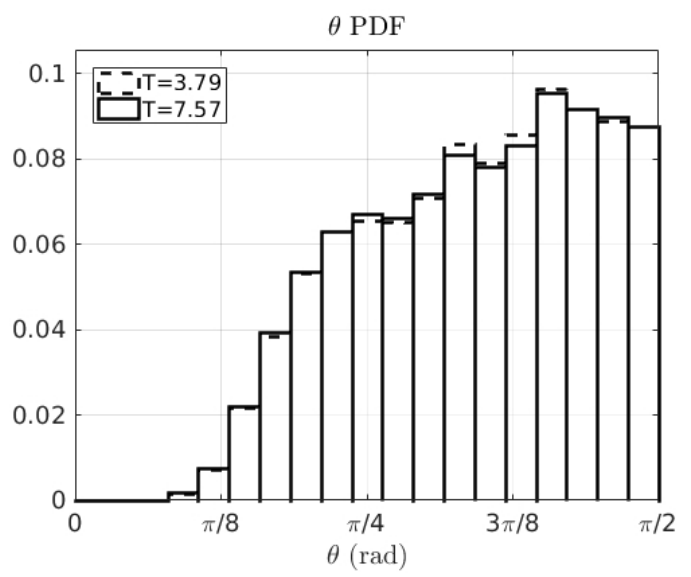

Figure 12. PDF of $\theta$ for the $D V 2$ experiment computed at $t=3.79$ (dashed line) and at $t=20$ (full line).

\section{Conclusions}

We have here proposed a new definition and a new computational framework to determine hyperbolic structures in a two-dimensional flow based on covariant Lyapunov vectors. CLVs are covariant with the dynamics, invariant for temporal inversion and norm independent. These vectors are the natural mathematical entity to probe the asymptotic behavior of the tangent space of a dynamical system. All these properties allow an exploration of the spatial structures of the flow, which cannot be done using the Lyapunov vectors bases due to their orthogonality.

CLVs are related to the contraction and expansion directions passing through a point of the tangent space, and the angle between them can be thus considered as a measure of the hyperbolicity of the system. This information can be summarized in a scalar field, the angle $\theta$, between the CLVs referring to the initial grid conditions, and used to define hyperbolic structures. The structures identified with the isolines of this field, characterized by $\theta=\pi / 2$, are called hyperbolic covariant coherent structures. These patterns are the most repelling or attracting pointwise, in terms of the asymptotic behavior of tracer particles, with respect to nearby structures at a given time. In terms of practical applications, this has important consequences, as it will provide an indicator for the long time transport of passive tracers such as for example oil spills in the ocean.

CLVs, and the correspondent $\theta$ field, have been computed for three numerical examples to compare how the behavior of the particle tracer near HLCSs, highlighted by the FTLEs, can change asymptotically in time. The three examples include a Hamiltonian autonomous system and two non-autonomous systems that are bounded or periodic. For all these examples, it is possible to compute CLVs, HCCSs and compare them with the HLCSs. Since the FTLEs tend to converge to LEs and lose their dependence on the initial conditions, the angle between the CLVs could give more detailed information about possible structures that can emerge from the flow. This feature has been highlighted in particular for the Hamiltonian autonomous system, in which $\theta$ is able to detect the central barrier in contrast with the FTLE field, and in the Bickley jet, in which the FTLEs converge to the same value in the jet region, and it is not possible to see any kind of particular finest structure. The use of $\theta$ provides information on the structures appearing at each time of the evolution of the flow, and the three examples underline that the HCCSs did not always correspond to HLCSs and vice versa. Therefore, particle tracers, such as chlorophyll or oil in water, can be maximally attracted or repelled by some HLCSs, but if we consider a different time interval and in particular the asymptotic behavior of these particles, we can obtain a distribution that is completely different. Note that, in practice, the asymptotic time length can be considered as the time taken by two random initial bases to converge to the same BLVs basis.

It should be noticed that, while no fluxes can be present across the HLCSs, the same does not necessarily hold for all the structures appearing characterized by $\theta=\pi / 2$. HCCSs can be found for 
every instant of time, but they give asymptotic information about the behavior of the particles tracer near the HCCS at that instant of time, and for this reason, the zero flux requirement of the HLCSs is not necessary. HCCSs are not necessarily a barrier, and their meaning is different from the one of HLCSs.

For the three examples considered, we have also computed HLCSs with the geodesic theory using the LCS tool [66] (not shown). The results are in agreement with the discussion above. Looking at asymptotic time, it is still possible to find particular structures emerging from flow, but clearly, these structures do not necessarily correspond to HLCSs computed for a particular time interval.

For the two non-autonomous systems, we have considered the evolution of the PDFs of the $\theta$ field and the evolution of its first four moments. For the Bickley jet, the probability distribution of the angle is stationary in time, and so its moments, but for the double gyre, it is possible to appreciate a small variation in time for the PDF. The information deriving from the evolution of the $\theta$ field, related to the variation of the strength of the hyperbolicity field, could be used to characterize the dynamical mixing of the system.

Finally, future studies will have to address the detection of hyperbolic structures beyond analytical systems, i.e., for two-dimensional turbulent flows. This will be particularly interesting for flows at the transition between balance and lack of balance [69-72], where the detection of HCCSs can shed light both on the structure of mixing and on the forward cascade of energy to dissipation, or the lack of thereof. In particular, the evolution of the moments of the PDF of $\theta$ could be used to define an index of dynamical mixing for the system under study. Particularly interesting will be the behavior of the HCCSs in the presence of intermittency. We can conjecture that in particular cases, such as, e.g., the merging of two vortices, the instantaneous structures underlined by the HCCS can give reliable information about the asymptotic tracer dynamics, that is the dynamics after the merging event. This is however left for future studies.

Acknowledgments: We would like to thank two anonymous referees for comments that helped to improve the manuscript. This study was partially funded by the Research Grant DFG1740. GB was partially funded also by the Research Grants DFG TRR181 and DFG BA 5068/8-1. The authors would also like to thanks Sergiy Vasylkevych and Sebastian Schubert for interesting discussions on the subject.

Author Contributions: G.C. and G.B.conceived and designed the experiments. G.C. performed the experiments. G.C. and G.B. analyzed the data. G.C. and G.B. wrote the paper.

Conflicts of Interest: The authors declare no conflict of interest. The founding sponsors had no role in the design of the study; in the collection, analyses or interpretation of data; in the writing of the manuscript; nor in the decision to publish the results.

\section{Appendix A. Description of the Algorithm}

The algorithm for the identification of CLVs is based on five different phases:

1. Initialization (T1): This preliminary step is used to find the initial backward Lyapunov vector bases $\left\{\mathbf{1}_{1}^{-}(t), \mathbf{l}_{2}^{-}(t)\right\}$ for a whole set of initial conditions [73]. A set of initial conditions $\left\{\mathbf{x}_{\mathbf{0}}\right\} \in D$ and two sets of initial orthonormal random bases are defined in the tangent spaces at every point at time $t_{0}$. The second set is necessary to check the convergence to the backward Lyapunov vector bases. The initial conditions and the random bases are evolved respectively with Equations (1) and (6) until the convergence of the two bases is reached with the desired accuracy. The convergence toward the Lyapunov vectors is typically exponential in time [51,74]. At every time step, for every initial condition, the evolved vectors are stored as a column of a matrix that is decomposed with a QRdecomposition. The last passage is implemented in order to find the new orthogonal basis at every time step and the upper triangular matrix containing the coefficients that allow one to express the old basis in terms of the new one.

2. Forward transition (T2): The backward Lyapunov bases are evolved from time $t$ to $t^{\prime}$. The evolution is done with the help of Equations (1) and (6) and the $Q R$ decomposition for every evolution step. We indicate with $\mathbf{X}\left(t_{k}\right)$ the matrix for which columns contain the new bases at time $t_{k}$ and with $\mathbf{R}\left(t_{k-1}, t_{k}\right)$ the correspondent upper triangular matrix. During this step, 
both the local Lyapunov bases and upper triangular matrices are stored. The diagonal elements $\left(\mathbf{R}\left(t_{k-1}, t_{k}\right)\right)_{i i}$ of the upper triangular matrices give information about the local growth rates of the basis vectors at a given time $t_{k}$, and they are used to compute the FTLEs as a time average:

$$
\lambda_{i}=\frac{1}{t^{\prime}-t} \sum_{k=0}^{N-1} \log \left(\mathbf{R}\left(t_{k-1}, t_{k}\right)\right)_{i i}
$$

where $N$ time steps are considered between $t$ and $t^{\prime}$. If the LEs exist, Equation (A1) will converge to them for a sufficiently long evolution. It is worth noting that sometimes the FTLEs are computed using a different method, that is using the so-called Cauchy-Green Tensor (CGT) defined as:

$$
\mathbf{G}\left(t, t^{\prime}\right)=\mathbf{F}\left(t, t^{\prime}\right)^{\top} \mathbf{F}\left(t, t^{\prime}\right) \text {. }
$$

This operator is also known as the deformation tensor, whose eigenvalues $\mu_{i}\left(t_{0}, t\right)$ and eigenvectors $\xi_{i}\left(t_{0}, t\right)$ satisfy:

$$
\begin{aligned}
& \mathbf{G}\left(t, t^{\prime}\right) \xi_{i}\left(t, t^{\prime}\right)=\mu_{i}\left(t, t^{\prime}\right) \xi_{i}\left(t, t^{\prime}\right), \\
& \mu_{1}\left(t, t^{\prime}\right)>\mu_{2}\left(t, t^{\prime}\right)>0, \\
& \xi_{1}\left(t, t^{\prime}\right) \perp \xi_{2}\left(t, t^{\prime}\right) .
\end{aligned}
$$

From a geometric point of view, a set of initial conditions corresponding to the unit sphere is mapped by the dynamics into an ellipsoid, with the principal axis aligned in the direction of the eigenvectors of the CGT and with length determined by the correspondent eigenvalues. The eigenvalues of the CGT determine the FTLEs as:

$$
\lambda_{i}\left(t, t^{\prime}\right)=\frac{1}{2\left(t^{\prime}-t\right)} \log \left(\mu_{i}\left(t, t^{\prime}\right)\right),
$$

where the dependence to the starting position has here been suppressed. This second method for the computation of the FTLEs exhibits some problems; for example, if just one finite local growth rate is taken into account considering a large time interval, Equation (A4) tends to zero and not to the LEs. The first method should be preferred.

3. Forward dynamics (T3): In this step, the trajectories and the bases are further evolved from time $t^{\prime}$ to time $t^{\prime \prime}$ using Equations (1) and (6). This time interval should grant convergence, during the backward dynamic, to the CLVs. During this step, only the upper triangular matrices are stored and are used to continue the computation of the FTLEs using Equation (A4).

4. Backward transition (T4): In this step, random upper triangular matrices are generated for every point of the grid, $\mathbf{C}$. These matrices contain the expansion coefficients of a set of two generic vectors (expressed as the column of a matrix) in terms of the $L^{-}$bases. Using the stored matrices $\mathbf{R}$ of Step 3, these matrices are evolved backward in time, until time $t^{\prime}$, using the following relation:

$$
\mathbf{C}\left(t_{n}\right)=\mathbf{R}^{-1}\left(t_{n}, t_{n+1}\right) \mathbf{C}\left(t_{n+1}\right) \mathbf{D}\left(t_{n}, t_{n+1}\right),
$$

where $t_{n}$ and $t_{n+1}$ being the time step between $t^{\prime}$ and $t^{\prime \prime}$. This method uses all of the information contained in $\mathbf{R}$ and not just the diagonal part of the matrix. The $\mathbf{D}$ diagonal matrices contain the column norm of $\mathbf{C}$. Using Equation (A5), it is possible to show [51] that the generic vectors chosen will be aligned with the CLVs. Note that when a trajectory passes close to the tangency of an invariant manifold, the matrices $C$ can be ill-defined, and so, a small amount of noise on the diagonal element of these matrices or an average on the diagonal elements of the neighbor matrices is used to correct the problem.

5. Backward dynamics (T5): In this final part of the algorithm, Equation (A5) is used with the $\mathbf{R}$ matrices of Step 2 to evolve backward the upper triangular matrices $\mathbf{C}$, from time $t^{\prime}$ to time $t$. 
In this phase, the backward Lyapunov bases stored can be used to write the CLVs. The matrix containing in each column the different CLVs at a given point in space and time, $\mathbf{W}$, can thus be written as:

$$
\mathbf{W}\left(t_{n}\right)=\mathbf{C}\left(t_{n}\right) \mathbf{X}\left(t_{n}\right) \text {. }
$$

For a two-dimensional system, the algorithm could be optimized making use of (18). One can follow the first step (T1) of the previous algorithm to find the convergence toward the backward Lyapunov bases in the time interval $\left[t_{0}, t\right]$. After this first step, it is possible to carry out the evolution of the vectors, as in the second step (T2) during the time interval $\left[t, t^{\prime}\right]$, without saving the triangular matrices. In the same way, it is possible to repeat the step (T1), but for a backward evolution during the time interval $\left[t^{\prime \prime}, t^{\prime}\right]$ to find the forward Lyapunov bases and then continue the backward evolution as in the step (T2) during the time interval $\left[t^{\prime}, t\right]$. At this stage, it is possible to consider directly the first backward Lyapunov vectors and the second forward Lyapunov vectors in the time interval $\left[t, t^{\prime}\right]$ as the CLVs. In this algorithm, there are just four steps and not five as in the one presented above, but for two times, one has to consider the convergence step (T1), which is more time consuming with respect to Step (T4) or (T5). It should be noted that in this algorithm, the forward and the backward evolutions could be done in parallel. The comparison between this algorithm and the one used for this study is left for future studies.

\section{Appendix B. Technical Details of the Numerical Examples}

\section{Appendix B.1. Hamiltonian System}

For the simple Hamiltonian flow, we consider the domain $x=[0.2,4], y=[-0.5,0.5]$, a resolution of $300 \times 300$ grid points and a time step $d t=0.01$. The CLVs are computed just for $t=5$, so the phases $\mathrm{T} 2$ and $\mathrm{T} 5$ include just a few time steps. The forward and backward evolution is done in the time interval $T=[5,10]$. From $t=0$ to $t=5$, the algorithm passes through the initialization phase (see Appendix A) to find the backward Lyapunov vectors bases. In Figure A1 is shown the convergence for the spatial average of the scalar product between the starting random bases chosen.

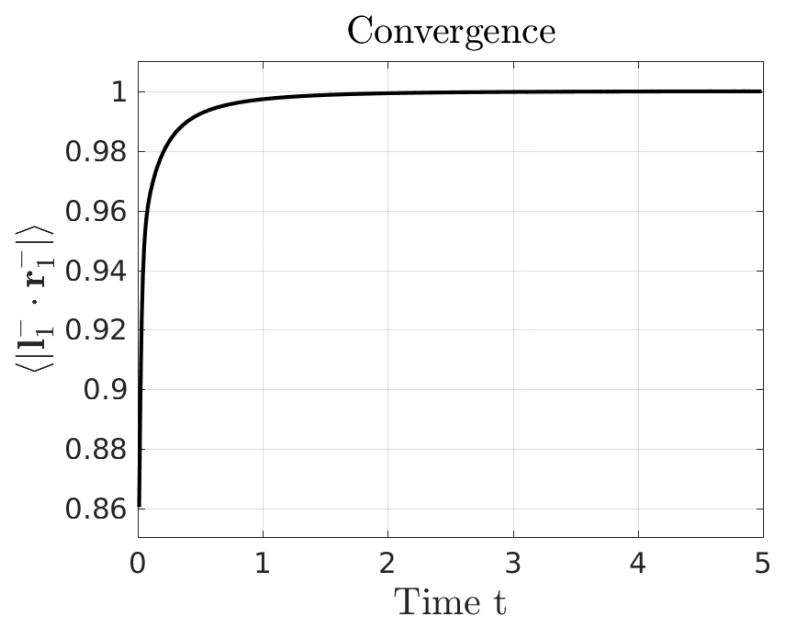

Figure A1. Convergence of the averaged scalar product for the two random bases chosen for the initialization of the numerical algorithm for the Hamiltonian system Equation (27).

\section{Appendix B.2. Double Gyre}

The parameters used for the double gyre example are $A=0.1, \epsilon=0.1$ and $\omega=\pi / 5$. The spatial domain is $x=[0,2], y=[0,1]$, with spatial resolution of $400 \times 200$ points and time step $d t=0.02$. 
Two experiments, $D V 1$ and $D V 2$, involving different CLVs' computational time intervals are considered and summarized in Table A1.

Table A1. The five temporal windows of the numerical algorithm used for the two experiments DV1 and $D V 2$.

\begin{tabular}{cccccc}
\hline & T1 & T2 & T3 & T4 & T5 \\
\hline$D V 1$ & $0 \rightarrow 5$ & $5 \rightarrow 10$ & $10 \rightarrow 15$ & $10 \leftarrow 15$ & $5 \leftarrow 10$ \\
$D V 2$ & $0 \rightarrow 10$ & $10 \rightarrow 20$ & $20 \rightarrow 30$ & $20 \leftarrow 30$ & $10 \leftarrow 20$ \\
\hline
\end{tabular}

During the initial phase of the numerical algorithm for the calculation of the CLVs, the average of the scalar product of the two initial random bases does not converge exactly to one (not shown here). This is because near the central regions of the two vortices of the double gyre, the expansion and contraction directions are not well defined, the CLVs become tangent to each other and their separation is difficult to attain.

\section{Appendix B.3. Bickley Jet}

The parameters used for this example are $U=62.66, c_{2}=0.205 U, c_{3}=0.461 U, L_{y}=1.77 \times 10^{6}$, $\epsilon_{2}=0.04, \epsilon_{3}=0.3, L_{x}=6.371 \pi \times 10^{6}, k_{n}=2 \pi n / L_{x}$. The spatial domain considered is $x=\left[0, L_{x}\right]$, $y=[-2.25,2.25] L_{y}$, with a resolution of $500 \times 250$ grid points and a time step of $d t=1800$. Two different time windows evolutions are considered, experiments $B J 1$ and $B J 2$, and summarized in Table A2.

Table A2. The five temporal windows of the algorithm used for the two experiments BJ1 and BJ2. Times have been rescaled with $L_{x} / U$.

\begin{tabular}{cccccc}
\hline & T1 & T2 & T3 & T4 & T5 \\
\hline BJ1 & $0 \rightarrow 1.89$ & $1.89 \rightarrow 3.79$ & $3.79 \rightarrow 5.68$ & $3.79 \leftarrow 5.68$ & $1.89 \leftarrow 3.79$ \\
BJ2 & $0 \rightarrow 3.79$ & $3.79 \rightarrow 7.57$ & $7.57 \rightarrow 11.36$ & $7.57 \leftarrow 11.36$ & $3.79 \leftarrow 7.57$ \\
\hline
\end{tabular}

\section{References}

1. Dombre, T.; Frisch, U.; Greene, J.M.; Hénon, M.; Mehr, A.; Soward, A.M. Chaotic streamlines in the ABC flows. J. Fluid Mech. 1986, 167, 353-391.

2. Haller, G. Lagrangian coherent structures. Ann. Rev. Fluid Mech. 2015, 47, 137-162.

3. Kelley, D.H.; Allshouse, M.R.; Ouellette, N.T. Lagrangian coherent structures separate dynamically distinct regions in fluid flows. Phys. Rev. E 2013, 88, doi:10.1103/PhysRevE.88.013017.

4. Chian, A.C.L.; Rempel, E.L.; Aulanier, G.; Schmieder, B.; Shadden, S.C.; Welsch, B.T.; Yeates, A.R. Detection of coherent structures in photospheric turbulent flows. Astrophys. J. 2014, 786, doi:10.1088/0004-637X/786/1/51.

5. Pierrehumbert, R.T.; Yang, H. Global chaotic mixing on isentropic surfaces. J. Atmos. Sci. 1993, 50, $2462-2480$.

6. Doerner, R.; Hübinger, B.; Martienssen, W.; Grossmann, S.; Thomae, S. Stable manifolds and predictability of dynamical systems. Chaos Solitons Fractals 1999, 10, 1759-1782.

7. Haller, G.; Yuan, G. Lagrangian coherent structures and mixing in two-dimensional turbulence. Phys. $D$ 2000, 147, $352-370$.

8. Haller, G. Distinguished material surfaces and coherent structures in three-dimensional fluid flows. Phys. D 2001, 149, $248-277$.

9. Lapeyre, G. Characterization of finite-time Lyapunov exponents and vectors in two-dimensional turbulence. Chaos 2002, 12, 688-698.

10. Shadden, S.C.; Lekien, F.; Marsden, J.E. Definition and properties of Lagrangian coherent structures from finite-time Lyapunov exponents in two-dimensional aperiodic flows. Phys. D 2005, 212, $271-304$.

11. Lekien, F.; Shadden, S.C.; Marsden, J.E. Lagrangian coherent structures in n-dimensional systems. J. Math. Phys. 2007, 48, doi:10.1063/1.2740025. 
12. Sulman, M.H.M.; Huntley, H.S.; Lipphardt, B.L., Jr.; kirwan, A.D., Jr. Leaving flatland: Diagnostics for Lagrangian coherent structures in three-dimensional flows. Phys. D 2013, 258, 77 - 92.

13. Rypina, I.I.; Brown, M.G.; Beron-Vera, F.J.; Koçak, H.; Olascoaga, M.J.; Udovydchenkov, I.A. On the Lagrangian dynamics of atmospheric zonal jets and the permeability of the stratospheric Polar Vortex. J. Atmos. Sci. 2007, 64, 3595-3610.

14. Rypina, I.I.; Pratt, L.J.; Pullen, J.; Levin, J.; Gordon, A.L. Chaotic Advection in an archipelago. J. Phys. Oceanogr. 2010, 40, 1988-2006.

15. Beron-Vera, F.J.; Olascoaga, M.J.; Goni, G.J. Oceanic mesoscale eddies as revealed by Lagrangian coherent structures. Geophys. Res. Lett. 2008, 35, doi:10.1029/2008GL033957.

16. Waugh, D.W.; Abraham, E.R. Stirring in the global surface ocean. Geophys. Res. Lett. 2008, 35, doi:10.1029/2008GL035526.

17. Bettencourt, J.H.; López, C.; Hernández-García, E. Oceanic three-dimensional Lagrangian coherent structures: A study of a mesoscale eddy in the Benguela upwelling region. Ocean Model. 2012, 51, 73 - 83.

18. Harrison, C.S.; Glatzmaier, G.A. Lagrangian coherent structures in the California Current System, sensitivities and limitations. Geophys. Astrophys. Fluid Dyn. 2012, 106, 22-44.

19. Waugh, D.W.; Keating, S.R.; Chen, M.L. Diagnosing ocean stirring: comparison of relative dispersion and finite-time Lyapunov exponents. J. Phys. Oceanogr. 2012, 42, 1173-1185.

20. Mukiibi, D.; Badin, G.; Serra, N. Three-dimensional chaotic advection by mixed layer baroclinic instabilities. J. Phys. Oceanogr. 2016, 46, 1509-1529.

21. Joseph, B.; Legras, B. Relation between kinematic boundaries, stirring, and barriers for the Antarctic polar vortex. J. Atmos. Sci. 2002, 59, 1198-1212.

22. D’Ovidio, F.; Fernández, V.; Hernández-García, E.; López, C. Mixing structures in the Mediterranean Sea from finite-size Lyapunov exponents. Geophys. Res. Lett. 2004, 31, doi:10.1029/2004GL020328.

23. Cencini, M.; Vulpiani, A. Finite size Lyapunov exponent: Review on applications. J. Phys. A 2013, 46, doi:10.1088/1751-8113/46/25/254019.

24. Karrasch, D.; Haller, G. Do finite-size Lyapunov exponents detect coherent structures? Chaos 2013, 23, doi:10.1063/1.4837075.

25. Karrasch, D. Attracting Lagrangian coherent structures on Riemannian manifolds. Chaos 2015, 25, doi:10.1063/1.4928451.

26. Mancho, A.M.; Wiggins, S.; Curbelo, J.; Mendoza, C. Lagrangian descriptors: A method for revealing phase space structures of general time dependent dynamical systems. Commun. Nonlinear Sci. Numer. Simul. 2013, $18,3530-3557$.

27. Lopesino, C.; Balibrea-Iniesta, F.; García-Garrido, V.; Wiggins, S.; Mancho, A. A theoretical framework for Lagrangian descriptors. Int. J. Bifurc. Chaos 2017, 27, doi:10.1142/S0218127417300014.

28. Vortmeyer-Kley, R. ; Gräwe, U. ;Feudel, U. Detecting and tracking eddies in oceanic flow fields: A Lagrangian descriptor based on the modulus of vorticity. Nonlin. Process. Geophys. 2016, 23, 159-173.

29. Froyland, G.; Lloyd, S.; Santitissadeekorn, N. Coherent sets for nonautonomous dynamical systems. Phys. D 2010, 239, 1527-1541.

30. Thiffeault, J.L. Braids of entangled particle trajectories. Chaos 2010, 20, doi:10.1063/1.3262494

31. Mundel, R.; Fredj, E.; Gildor, H.; Rom-Kedar, V. New Lagrangian diagnostics for characterizing fluid flow mixing. Phys. Fluids 2014, 26, doi:10.1063/1.4903239.

32. Skokos, C.; Bountis, T.; Antonopoulos, C. Geometrical properties of local dynamics in Hamiltonian systems: The Generalized Alignment Index (GALI) method. Phys. D 2007, 231, 30-54.

33. Cincotta, P.M.; Simo, C. Simple tools to study global dynamics in non-axisymmetric galactic potentials. Astron. Astrophys. Suppl. 2000, 147, 205-228.

34. Szezech, J.; Schelin, A.; Caldas, I.; Lopes, S.; Morrison, P.; Viana, R. Finite-time rotation number: A fast indicator for chaotic dynamical structures. Phys. Lett. A 2013, 377, 452-456.

35. Szezech, J.D., Jr.; Caldas, I.L.; Lopes, S.R.; Morrison, P.J.; Viana, R.L. Effective transport barriers in nontwist systems. Phys. Rev. E 2012, 86, doi:10.1103/PhysRevE.86.036206.

36. Szezech, J.D., Jr.; Caldas, I.L.; Lopes, S.R.; Viana, R.L.; Morrison, P.J. Transport properties in nontwist area-preserving maps. Chaos 2009, 19, doi:10.1063/1.3247349.

37. Haller, G. A variational theory of hyperbolic Lagrangian Coherent Structures. Phys. D 2011, 240, 574-598. 
38. Farazmand, M.; Haller, G. Erratum and addendum to A variational theory of hyperbolic Lagrangian coherent structures [Phys. D 240 (2011) 574-598]. Phys. D 2012, 241, 439-441.

39. Karrasch, D. Comment on A variational theory of hyperbolic Lagrangian coherent structures [Phys. D 240 (2011) 574-598]. Phys. D 2012, 241, 1470-1473.

40. Farazmand, M.; Haller, G. Computing Lagrangian coherent structures from their variational theory. Chaos 2012, 22, doi:10.1063/1.3690153.

41. Farazmand, M.; Haller, G. Attracting and repelling Lagrangian coherent structures from a single computation. Chaos 2013, 23, doi:10.1063/1.4800210.

42. Blazevski, D.; Haller, G. Hyperbolic and elliptic transport barriers in three-dimensional unsteady flows. Phys. D 2014, 273-274, 46-62.

43. Haller, G.; Beron-Vera, F.J. Geodesic theory of transport barriers in two-dimensional flows. Phys. D 2012, 241, 1680-1702.

44. Farazmand, M.; Blazevski, D.; Haller, G. Shearless transport barriers in unsteady two-dimensional flows and maps. Phys. D 2014, 278-279, 44-57.

45. Serra, M.; Haller, G. Objective Eulerian coherent structures. Chaos 2016, 26, doi:10.1063/1.4951720.

46. Serra, M.; Haller, G. Forecasting long-lived Lagrangian vortices from their objective Eulerian footprints. J. Fluid Mech. 2017, 813, 436-457.

47. Serra, M.; Haller, G. Efficient computation of null geodesics with applications to coherent vortex detection. Proc. R. Soc. Lond. A Math. Phys. Eng. Sci. 2017, 473, doi:10.1098/rspa.2016.0807.

48. Oseledec, V.I. A multiplicative ergodic theorem: Lyapunov characteristic numbers for dynamical systems. Trans. Mosc. Math. Soc. 1968, 19, 197-231.

49. Ruelle, D. Ergodic theory of differentiable dynamical systems. Publ. Math. IHES 1979, 50, $27-58$.

50. Wolfe, C.L.; Roger M., S. An efficient method for recovering Lyapunov vectors from singular vectors. Tellus $A$ 2007, 59, 355-366.

51. Ginelli, F.; Poggi, P.; Turchi, A.; Chaté, H.; Livi, R.; Politi, A. Characterizing dynamics with covariant Lyapunov vectors. Phys. Rev. Lett. 2007, 99, doi:10.1103/PhysRevLett.99.130601.

52. Froyland, G.; Hüls, T.; Morriss, G.P.; Watson, T.M. Computing covariant Lyapunov vectors, Oseledets vectors, and dichotomy projectors: A comparative numerical study. Phys. D 2013, 247, 18-39.

53. Bosetti, H.; Posch, H.A. Covariant Lyapunov vectors for rigid disk systems. Chem. Phys. 2010, 375, $296-308$.

54. Xu, M.; Paul, M.R. Covariant Lyapunov vectors of chaotic Rayleigh-Bénard convection. Phys. Rev. E 2016, 93, doi:10.1103/PhysRevE.93.062208.

55. Wolfe, C.L.; Samelson, R.M. Singular vectors and time-dependent normal modes of a baroclinic wave-mean oscillation. J. Atmos. Sci. 2008, 65, 875-894.

56. Schubert, S.; Lucarini, V. Covariant Lyapunov vectors of a quasi-geostrophic baroclinic model: Analysis of instabilities and feedbacks. Q. J. R. Meteor. Soc. 2015, 141, 3040-3055.

57. Schubert, S.; Lucarini, V. Dynamical analysis of blocking events: spatial and temporal fluctuations of covariant Lyapunov vectors. Q. J. R. Meteor. Soc. 2016, 142, 2143-2158.

58. Vannitsem, S.; Lucarini, V. Statistical and dynamical properties of covariant Lyapunov vectors in a coupled atmosphere-ocean model-multiscale effects, geometric degeneracy, and error dynamics. J. Phys. A 2015, 49, doi:10.1088/1751-8113/49/22/224001.

59. Yang, H.L.; Radons, G. When can one observe good hydrodynamic Lyapunov modes? Phys. Rev. Lett. 2008, 100, doi:10.1103/PhysRevLett.100.024101.

60. Yang, H.L.; Radons, G. Comparison between covariant and orthogonal Lyapunov vectors. Phys. Rev. E 2010, 82, 10.1103/PhysRevE.82.046204.

61. Kuptsov, P.V.; Parlitz, U. Theory and computation of covariant Lyapunov vectors. J. Nonlinear Sci. 2012, 22, 727-762.

62. Sala, M.; Manchein, C.; Artuso, R. Estimating hyperbolicity of chaotic bidimensional maps. Int. J. Bifurc. Chaos 2012, 22, doi:10.1142/S0218127412502173.

63. Ginelli, F.; Chate, H.; Livi, R.; Politi, A. Covariant Lyapunov vectors. J. Phys. A 2013, 46, doi:10.1088/1751-8113/46/25/254005.

64. D’Ovidio, F.; Shuckburgh, E.; Legras, B. Local mixing events in the upper troposphere and lower stratosphere. Part I: Detection with the Lyapunov Diffusivity. J. Atmos. Sci. 2009, 66, 3678-3694.

65. Eckmann, J.P.; Ruelle, D. Ergodic theory of chaos and strange attractors. Rev. Mod. Phys. 1985, 57, 617-656. 
66. Onu, K.; Huhn, F.; Haller, G. LCS Tool: A computational platform for Lagrangian coherent structures. J. Comput. Sci. 2015, 7, 26-36.

67. Del Castillo-Negrete, D.; Morrison, P.J. Chaotic transport by Rossby waves in shear flow. Phys. Fluids A 1993, 5, 948-965.

68. Beron-Vera, F.J.; Olascoaga, M.J.; Brown, M.G.; Koçak, H.; Rypina, I.I. Invariant-tori-like Lagrangian coherent structures in geophysical flows. Chaos 2010, 20, doi:10.1063/1.3271342.

69. Badin, G.; Tandon, A.; Mahadevan, A. Lateral mixing in the pycnocline by baroclinic mixed layer eddies. J. Phys. Oceanogr. 2011, 41, 2080-2101.

70. Badin, G. On the role of non-uniform stratification and short-wave instabilities in three-layer quasi-geostrophic turbulence. Phys. Fluids 2014, 26, doi:10.1063/1.4895590.

71. Badin, G. Surface semi-geostrophic dynamics in the ocean. Geophys. Astro. Fluid Dyn. 2013, 107, 526-540.

72. Ragone, F.; Badin, G. A study of surface semi-geostrophic turbulence: Freely decaying dynamics. J. Fluid Mech. 2016, 792, 740-774.

73. Benettin, G.; Galgani, L.; Giorgilli, A.; Strelcyn, J.M. Lyapunov Characteristic Exponents for smooth dynamical systems and for hamiltonian systems; A method for computing all of them. Part 2: Numerical application. Meccanica 1980, 15, 21-30.

74. Goldhirsch, I.; Sulem, P.L.; Orszag, S.A. Stability and Lyapunov stability of dynamical systems: A differential approach and a numerical method. Phys. D 1987, 27, 311-337.

(C) 2017 by the authors. Licensee MDPI, Basel, Switzerland. This article is an open access article distributed under the terms and conditions of the Creative Commons Attribution (CC BY) license (http://creativecommons.org/licenses/by/4.0/). 